\title{
e-Phaïstos
}

e-Phaïstos Revue d'histoire des techniques / Journal of the history of technology

VIII-2 | 2020

Aviation : émergence d'un complexe technique, 1900-1930

\section{Schneider, les alliages légers et l'aviation} (1916-1939)

Schneider, Light Alloys and Aviation (1916-1939)

Jean-Philippe Passaqui

\section{OpenEdition}

Journals

Édition électronique

URL : https://journals.openedition.org/ephaistos/8111

DOI : 10.4000/ephaistos.8111

ISSN : 2552-0741

Éditeur

IHMC - Institut d'histoire moderne et contemporaine (UMR 8066)

Référence électronique

Jean-Philippe Passaqui, « Schneider, les alliages légers et l'aviation (1916-1939) », e-Phaïstos [En ligne], VIII-2 | 2020, mis en ligne le 27 octobre 2020, consulté le 17 septembre 2021. URL : http://

journals.openedition.org/ephaistos/8111 ; DOI : https://doi.org/10.4000/ephaistos.8111

Ce document a été généré automatiquement le 17 septembre 2021.

Tous droits réservés 


\title{
Schneider, les alliages légers et l'aviation (1916-1939)
}

Schneider, Light Alloys and Aviation (1916-1939)

\author{
Jean-Philippe Passaqui
}

1 La Première Guerre mondiale a considérablement modifié la trajectoire industrielle de certaines entreprises françaises ${ }^{1}$. En fonction des besoins de la Défense nationale, elles ont été amenées à fabriquer des objets, des matériels, très éloignés de leurs pratiques courantes. Par exemple, l'organisation productive des constructeurs automobiles s'est avérée adaptée à la demande massive d'obus. Mais pour certaines grandes entreprises métallurgiques du Centre de la France, les commandes de la Défense nationale correspondent à un savoir-faire déjà maîtrisé. Châtillon-Commentry, avec son usine Saint-Jacques, à Montluçon, Marine-Homécourt et son site de Saint-Chamond, ainsi que Schneider, identifié au Creusot, entrent dans la guerre munis d'un outillage puissant, composé d'équipements de forgeages, des presses hydrauliques et des marteaux pilons en l'occurrence, qui sont essentiels au moment de fabriquer des canons, des obus et des blindages ; le glaive et la cuirasse en quelque sorte.

2 Par contre, ces entreprises peinent à adapter leurs ateliers aux commandes standardisées reposant sur des volumes considérables. Elles ne disposent pas de la même aptitude à répondre aux besoins de l'artillerie française en obus de petits et moyens calibres, productions qu'elles abandonnent au cours du conflit. À l'inverse, elles possèdent des laboratoires de recherches, de métrologie et d'essais, de véritables bureaux d'études, qui sont en mesure de concevoir de nouveaux objets techniques, dont les champs de bataille de la Première Guerre mondiale ont constitué un terrain d'expérimentation. Ainsi, avec la guerre vient le temps des études relatives aux véhicules blindés et chenillés. Pendant que Schneider et Cie assemblent, sur leurs différents sites, le char CA1, Saint-Chamond tente de mettre au point un matériel équivalent. La diversité caractérise les équipements produits.

3 Souvent, sous l'effet des nombreuses sollicitations du ministère de la Guerre, un effort de recherches sans précédent est mené. Il s'appuie sur ce que ce que les entreprises savent déjà produire. À ce titre, la conception du char Schneider déployé par l'Artillerie 
spéciale s'apparente à une greffe d'éléments dont l'entreprise maitrise la production, comme les tôles, les plaques et le canon, associés à des équipements, les chenilles et le moteur, pour lesquels elle organise une filiale, la SOMUA (Société d'outillage mécanique et d'usinage d'artillerie), sur laquelle nous reviendrons. Il s'agit alors d'un matériel hybride, associant les compétences de l'entreprise à un certain nombre d'éléments empruntés à d'autres objets techniques, comme les tracteurs. En tant que tel, le char Schneider n'apporte aucune nouveauté. Il se contente d'agréger des équipements connus (Passaqui 2018a). Dans sa première version, la seule produite en série, il ne constitue pas un objet de rupture technique. Mais les entreprises apprennent vite, car les retours d'expériences sont nombreux et si des tensions règnent entre les services techniques de l'armée et les industriels, il n'en demeure pas moins que les discussions finissent par porter leurs fruits. Elles conduisent à l'amélioration des matériels conçus dans l'urgence.

4 Un moment paraît plus particulièrement favorable aux convergences d'intérêts. Pendant les combats, les assauts et la progression de l'infanterie permettent parfois de belles prises d'objets techniques qui sont ensuite adressés aux industriels pour expérimentations, dans le but d'imiter ou de contrer. C'est le principe de la rétroingénierie. Les envois depuis le front pour expertise, essais et analyses constituent des accélérateurs d'appropriation de savoir-faire. Ils peuvent être à l'origine d'un transfert technique rapide et inespéré. C'est ce qui se produit quand des caisses de munitions allemandes, notamment celles qui approvisionnent les fusils antichars, sont expédiées au Creusot. Elles sont essayées pour connaître leur capacité de pénétration des tôles et plaques de protection, dans le dessein d'améliorer la qualité des blindages qui recouvrent les chars en cours d'assemblage. La trempe et autres traitements thermiques des plaques, ainsi que l'épaisseur des blindages additionnels sont adaptés aux résultats des épreuves de résistance réalisées à partir des matériels allemands (Passaqui 2018b). Les établissements Schneider peuvent améliorer la protection des véhicules chenillés, avant même leurs premiers engagements, au cours de l'offensive du Chemin des Dames, en avril 1917. Même si les combats révèlent d'autres faiblesses, comme le débit trop faible de la ventilation et la localisation des réservoirs dans une partie exposée du véhicule, le châssis, en lui-même, s'avère plus solide que ses équivalents britanniques.

5 Cet exemple démontre qu'à partir de 1914, le panel des objets techniques et matériels de guerre conçus et assemblés dans les usines Schneider dépasse largement le périmètre souvent mis en exergue : celui des canons de tous calibres. Depuis le décès d'Henri Schneider en 1898, une des caractéristiques de l'entreprise, d'ailleurs source de tensions internes (Bergeron 2004), réside dans sa capacité à s'associer à des initiatives industrielles très éloignées de ses métiers habituels. Son usine du Creusot sort fréquemment des activités qui ont fait sa réussite pour tenter de s'engager, souvent avec des déconvenues, dans des secteurs d'activité prometteurs. Il en découle, à la fin du XIX ${ }^{e}$ siècle, une tentative d'immixtion dans le domaine de l'électromécanique, qui aboutit à la création d'une vaste usine à Champagne-sur-Seine.

6 À la même époque, Eugène II Schneider envisage d'ériger un vaste complexe industriel à Sète, qui partirait des fours à coke et des hauts fourneaux pour, en définitive, recevoir des chantiers de constructions navales. L'expérience tourne au fiasco mais elle n'empêche pas l'entreprise de se lancer dans d'autres activités. L'intérêt porté à la 
fabrication de bateaux de guerre s'opère ensuite à partir des usines de Chalon-surSaône et de la filiale des Ateliers de la Gironde (d'Angio 2000).

7 Les établissements Schneider et Cie cherchent enfin à se diversifier dans la construction automobile, les bus en particulier. Ils suivent avec intérêt les progrès effectués dans le domaine et s'associent avec un ingénieur, Eugène Brillé, dans le but de créer un atelier de véhicules. Si le projet ne dépasse guère le stade semi-industriel, il connaît un nouvel élan avec la Première Guerre mondiale. Schneider et Cie se portent acquéreur des anciens établissements Farcot à Saint-Ouen à partir desquels ils organisent une filiale amenée à connaître d'importants développements : la SOMUA. Celle-ci est articulée dès l'origine selon un cadre composite, qui associe les contraintes du moment, fournir les armées, aux objectifs futurs. Cela passe notamment par la création d'un service capable de produire tout à la fois du matériel agricole, des automobiles et des autobus. Pendant la guerre, la SOMUA assemble essentiellement des canons de $155 \mathrm{~mm}$, les chars Schneider CA1, les tracteurs d'artillerie CD, ainsi que de nombreux chars légers Renault (Passaqui 2018a).

8 Engagés dans une logique de diversification tous azimuts tout en cherchant à préserver les principes de l'intégration industrielle, les établissements Schneider entament finalement une réflexion portant sur les constructions aéronautiques. De nouvelles fabrications militaires doivent être recherchées. La croissance de la production d'avions de combat pendant la guerre, les applications civiles qui semblent devoir en découler à moyen terme, sont autant de sujets dignes d'intérêt. L'engagement industriel dans l'aéronautique peut constituer une voie de reconversion, au moins partielle, qui semble tentante.

9 Ces questions étaient déjà suivies avant-guerre au titre de la veille technologique, mais une série de sollicitations viennent, à partir de 1916, relancer l'espoir de pouvoir se diversifier vers des productions de ce type. Au même moment, bien que mise en avant pour sa participation à l'effort de guerre, l'entreprise est confrontée à une situation inconfortable, qui la rend vulnérable. Elle est en train de perdre son statut de partenaire privilégié de la Guerre. Certains programmes emblématiques, comme celui du char moyen à tourelle dit CA3, sont abandonnés, malgré les engagements contractuels de l'État, tandis que l'industrialisation de son tracteur d'artillerie, dit tracteur $C D$, s'éternise et perd de son intérêt.

10 En dehors de ses matériels inclus dans le strict périmètre de l'artillerie, Schneider se voit, comme dans le cas de sa participation à la construction du char léger Renault, reléguée à la production de tôles ou au rôle de sous-traitant, par le biais de la SOMUA. C'est trop peu pour l'entreprise qui est confrontée à des incertitudes quant à l'évolution de son carnet de commandes (Beaud 1998). Pareille évolution n'est pas acceptable pour Eugène II Schneider, le gérant de l'entreprise fondée en 1837 par son grand-père, Eugène I, et son grand-oncle. Nous regarderons donc comment et sous quelles formes les établissements Schneider et Cie en sont venues à déployer leurs compétences pour devenir des acteurs de la production de matériels de guerre aériens.

11 L'entreprise dispose d'un savoir-faire métallurgique reconnu, sur lequel elle s'appuie pour compléter ses connaissances par rapport à des matériels dont elle découvre les caractéristiques. Elle entend les produire dans leur intégralité, en partant de l'élaboration du métal. Mais en définitive, les échecs répétés de cette stratégie débouchent, pendant l'entre-deux-guerres, sur un cantonnement des activités industrielles à la mise au point et à la production d'alliages légers, pour lesquels le 
savoir-faire accumulé dans le domaine des aciers spéciaux s'avère précieux, car transposable rapidement, sans avoir à multiplier les investissements dans un outillage et des machines à l'utilité incertaine.

\section{La découverte des matériaux destinés aux constructions aéronautiques}

12 Dans une entreprise qui a bâti sa réputation sur sa capacité à produire des aciers variés et d'excellente qualité, s'intéresser à une activité, les constructions aériennes, qui repose encore essentiellement sur du bois, de la colle et de la toile, peut susciter l'étonnement. Les services métallurgiques au sein des établissements Schneider et Cie servent d'abord à alimenter les ateliers situés en aval, générant une plus forte valeur ajoutée. L'entreprise s'est engagée à cette fin dans la mise au point d'aciers spéciaux, avec de multiples déclinaisons autour d'alliages d'acier avec des pourcentages significatifs de nickel, chrome et molybdène (Passaqui 2012). Elle n'a jamais vraiment cherché à se confronter à d'autres métaux. Par contre, pendant la Première Guerre mondiale, elle a relancé et augmenté les fabrications de plusieurs aciers spéciaux, afin de répondre aux besoins des constructions aériennes et de chars².

Bien qu'ayant peu de connaissances dans le domaine de l'aluminium, l'entreprise Schneider possède plusieurs caractéristiques dans ses champs de compétences qui plaident en sa faveur. Par exemple, elle s'appuie sur son laboratoire, un des plus anciens et des plus innovants. Créé en 1861, il a acquis, au cours des années 1880, une reconnaissance universelle, grâce aux découvertes majeures menées par certains ingénieurs. Parmi eux, Floris Osmond et Jean Werth se sont illustrés par leurs travaux très innovants sur la théorie cellulaire de l'acier qui ont constitué le point de départ des recherches métallographiques (Chezeau 2004, Chezeau 2006)33. À la même époque, toujours au Creusot, Charles Walrand a participé à l'adaptation d'une sole basique ${ }^{4}$ au four Martin. Cette excellence ne s'est pas émoussée et elle reste, en France, avec sa concurrente Commentry-Fourchambault et Decazeville, celle qui est la plus à même de déterminer et reproduire les caractéristiques d'un alliage (Passaqui 2020).

Son service de documentation organise une sorte de veille technologique à partir d'un dépouillement attentif de la presse technique européenne et américaine. Bien que l'aluminium soit une invention française, c'est surtout par le prisme des revues étrangères que l'entreprise se tient informée. Le contenu le plus essentiel est recopié, compilé, avant d'être diffusé au sein des services potentiellement concernés. Il se contente de réunir les avancées faites dans l'avant-guerre autour de l'aluminium, en diffusant les premiers alliages légers, et, en particulier, le Duralumin. Ce dernier présente en effet la particularité de réunir, d'après ses concepteurs et promoteurs, les qualités de légèreté de l'aluminium, ainsi que de résistance et de dureté de l'acier 5 . Schneider n'envisage pas de franchir le pas de la production, mais se place en situation de riposter au cas où l'émergence de ces matériaux entrerait en concurrence avec ses aciers.

15 En effet, les alliages légers de type Duralumin ${ }^{6}$ peuvent apparaitre comme des matériaux susceptibles de concurrencer certains de ses aciers spéciaux. Ils possèdent une légèreté bien supérieure, tout en ayant l'avantage de disposer des caractéristiques physiques, de résistance et de dureté, qui les rapprochent de celles de l'acier (Guiminot et Tissier 2015). À ce titre, dès le début du XXe siècle, avant même qu'il ne soit prévu de 
produire des alliages légers, l'entreprise en suit avec attention les premiers développements. L'usine du Creusot a pris et conservé l'habitude de scruter attentivement l'évolution des marchés métallurgiques. Elle cherche à ne pas subir à nouveau la surprise qu'a constituée la diffusion rapide des aciers Bessemer (Garçon 2020), au moment même où elle augmentait encore ses capacités de production de fer puddlé. La guerre n'interrompt pas cette habitude.

16 À côté des recherches dont les résultats sont immédiatement opératoires, comme dans le cas des tôles et plaques pour chars et autres véhicules blindés, des études sont engagées pour identifier les caractéristiques des matériaux de l'ennemi quand ils ne figurent pas encore dans les catalogues proposés par les industriels français. Déterminer la composition du métal dont sont faits les canons et les munitions de l'adversaire correspond aux champs de compétence du laboratoire du Creusot. Mais il est aussi sollicité en cas de prises de guerre plus originales, qui l'éloignent de ses activités habituelles. C'est dans ce cadre que se place une étude des marchés potentiels qu'offriraient les constructions aéronautiques. Elle laisse espérer qu'il serait possible de maîtriser l'ensemble de la filière, depuis la production de métal jusqu'à la réalisation d'objets techniques complets, à condition d'innover et de modifier la conception des avions, afin d'y incorporer une proportion beaucoup plus grande de métal, à la place du bois et de la toile.

17 C'est pourquoi, au cours de la Première Guerre mondiale, la question des alliages légers, présentée jusqu'à présent comme secondaire, est reconsidérée. L'entreprise ne se contente plus d'observer ; elle est partie prenante à plusieurs titres et devient un acteur de cette filière en pleine expansion. Pourtant, ses efforts nombreux et dans des directions très variées ont laissé peu de traces matérielles, ni même suscité de travaux, et ceci malgré l'abondance des archives. Comme l'engagement dans les constructions aériennes a été tardif, concentré sur les années 1917-1918, il n'a que rarement été poussé au-delà des études préliminaires. Mais pourquoi une entreprise reconnue dans le domaine des aciers qui constitue sa base technologique en vient-elle à focaliser son attention sur les alliages légers dont il constitue la base? Avant même de se poser la question, les établissements Schneider participent déjà à l'effort de guerre dans le domaine de la production d'avions.

18 Certaines parties des appareils relèvent de la compétence des métallurgistes. Le Creusot est engagé dans la production de quelques pièces d'acier, notamment celles destinées aux moteurs et à leurs supports. Pour les soupapes et les vilebrequins, l'excellence française dans les aciers spéciaux est d'ores et déjà confirmée. Les industriels du Centre de la France proposent aux ateliers de constructions mécaniques de nombreuses nuances d'aciers dédiés, ayant subi des essais chimiques et mécaniques répétés. Schneider fait partie des entreprises en mesure de proposer les aciers requis. L'usine du Creusot produit des bombes et, surtout, 531800 cylindres d'aviation au cours du conflit (Favre 2002) .

19 Elle fournit certaines pièces qui sont ébauchées au Creusot. Mais l'essentiel de son activité consiste à couler des lingots expédiés vers d'autres entreprises pour l'usinage de vilebrequins. En parallèle, le site d'Harfleur, créé en 1905 à proximité de son usine d'artillerie du Havre, connaît d'importants agrandissements. Il remporte un contrat pour assurer le montage d'un millier de moteurs d'avions type "Lorraine ${ }^{8}$ de 400 chevaux, dont les vilebrequins sont produits au Creusot. Il s'agit d'une pièce forgée 
soumise à de très fortes contraintes, qui ne peut être réalisée qu'à partir d'aciers dont les caractéristiques mécaniques sont déterminées avec précision.

Mais pendant la Première Guerre mondiale, le ciel n'est pas réservé qu'aux avions. Bien que les dirigeables aient montré dès leurs premiers engagements leur vulnérabilité, les armées allemandes continuent à les utiliser pendant toute la durée du conflit, à des fins de bombardements tactiques nocturnes, comme arme psychologique, mais aussi en appui de leur présence maritime. Dans la mesure où, dès la bataille de la Marne, l'aviation d'observation a démontré tout son intérêt mais aussi à la suite de la répétition d'accidents avant la guerre, la France n'a pas pris la même trajectoire que l'Allemagne. La question de l'emploi de dirigeables n'apparaît pas essentielle. Elle ne retient l'attention qu'à partir du moment où il devient nécessaire de couvrir des distances considérables que ne peuvent franchir les avions.

La traque effectuée par les sous-marins allemands en fournit l'impérieuse occasion. Elle désorganise les convois maritimes entre le Royaume-Uni, les États-Unis, d'une part, et la France, voire l'Italie, d'autre part. La question se pose donc surtout à partir de 1917, quand l'Allemagne se lance dans une guerre sous-marine à outrance. Les torpillages constituent une gêne pour le maintien des flux d'approvisionnements, non seulement par les tonnages coulés, mais aussi parce que certaines compagnies refusent de sacrifier leur flotte et l'envoient se réfugier dans l'océan pacifique. Le cas des pétroliers américains, au moment où l'armée française se motorise de plus en plus et consomme davantage de carburants, est caractéristique de la tension qui en découle.

Par conséquent, pour la Marine de guerre française, intensifier la détection et l'élimination des sous-marins allemands devient une priorité. Or, les moyens dont elle dispose ne sont pas adaptés à la mission. C'est pourquoi elle relance l'idée d'équiper la France de dirigeables qui auraient pour mission, non pas de harceler les troupes allemandes sur terre, mais bien de repérer les sous-marins ennemis afin de les détruire. Comme les compétences et surtout les acteurs industriels font défaut, il faut susciter en hâte la constitution d'entreprises qui n'ont pas encore l'envergure suffisante pour passer de la conception à la production de matériels de guerre aussi complexes par les dimensions qui les caractérisent mais aussi par l'hétérogénéité des matériaux qui les composent.

23 Pour parvenir rapidement à bonnes fins, les prises de guerre constituent une remarquable source d'informations. C'est pourquoi les Zeppelins détruits ou échoués en France font l'objet d'une expertise poussée, d'ailleurs largement médiatisée. Il s'agit de trouver le moyen de contrecarrer l'adversaire ou, le cas échéant, d'imiter ses réussites. Les usines du Creusot reçoivent quelques échantillons de leur structure métallique interne. Il s'agit d'alliages légers prélevés sur des points structurels particuliers des Zeppelins : certains profilés, les croisillons et des assemblages rivetés. Le Creusot a pour mission première d'en déterminer la composition ainsi que les caractéristiques physiques. Plusieurs séries d'échantillons permettent d'affiner les premiers résultats.

24 Le lot initial provient d'un Zeppelin, le LZ47, abattu à Revigny, le 21 février 1916, alors qu'il était en mission de bombardement sur les arrières des lignes françaises, à quelques heures du début de la bataille de Verdun (Alloitteau 1985) ${ }^{9}$. L'épisode connaît un certain retentissement car l'échec du raid de Zeppelins repousse la tentative de destruction d'un nœud ferroviaire français essentiel à l'acheminement des hommes et des moyens matériels. 


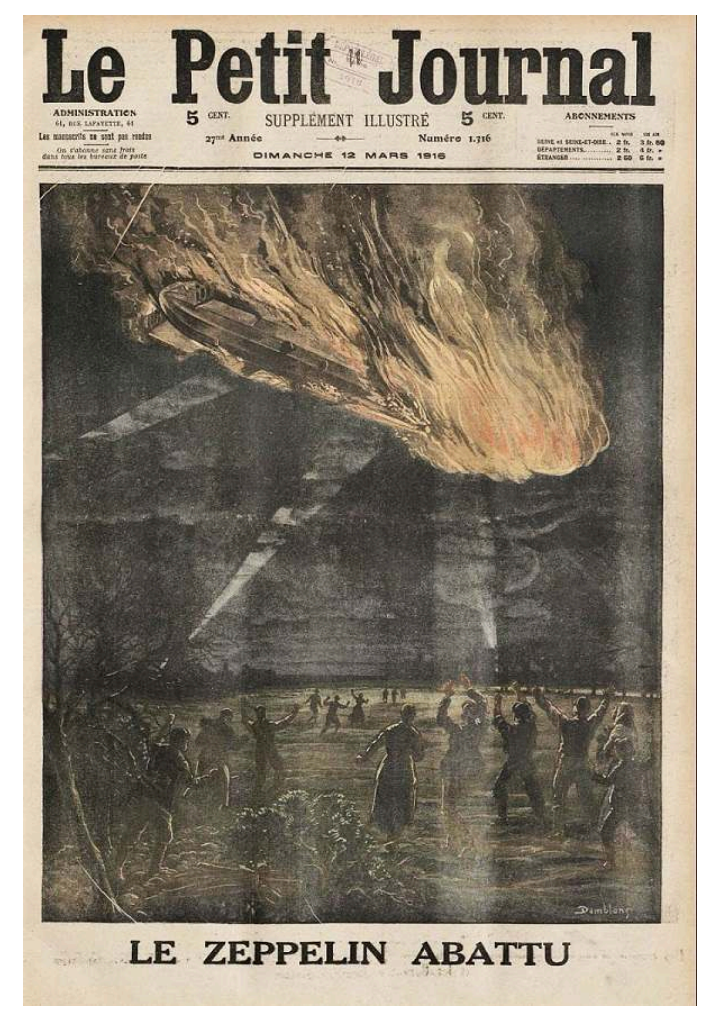

La destruction des Zeppelin fut largement mise en avant par les journaux pendant la Première Guerre mondiale. Ils étaient une source de terreur par le caractère spectaculaire de leurs raids et des destructions qu'ils provoquaient. Mais en retour, ils subissaient des pertes importantes qui rendaient ces raids risqués. Dans ce cas, il s'agit du LZ-77, détruit par la DCA française à Revigny-sur-Ornain, dans la Meuse, au moment du déclenchement de la bataille de Verdun. Sa dislocation limita l'exploitation des fragments métalliques de sa structure interne. Les usines du Creusot ne reçurent à ce titre que quelques échantillons.

Gravure du Petit Journal, supplément illustré, n¹316, 12 mars 1916, https://gallica.bnf.fr/ark:/12148/ bpt6k7172006.item

Mais le dirigeable, sans doute touché par l'artillerie française, s'est embrasé avant d'être pulvérisé à l'impact. Les restes sont en très mauvais état. Quelques fragments peuvent tout de même être analysés. Certains des profilés qui en sont retirés parviennent au laboratoire du Creusot. Leur réception intervient dans des circonstances peu favorables puisque ce service est confronté à un important surcroît d'activités. Mais aux contraintes du moment s'oppose la préparation du redéploiement d'après-guerre. Car si Schneider et Cie sont depuis plusieurs décennies reconnus pour la qualité de leurs aciers, ils entendent profiter de l'émergence, pendant la guerre, d'autres matériaux.

L'affaire est pilotée par Louis Bassal, le directeur de l'usine du Creusot. Il entend ne pas cantonner les travaux du laboratoire à une simple étude de la composition des restes du Zeppelin. Son projet est beaucoup plus ambitieux et engage l'entreprise vers des tentatives d'industrialisation des alliages légers: «Il ne vous échappe pas que nous chargeons ainsi un Ingénieur du Laboratoire d'une tâche qui est notablement distincte de ses attributions normales, mais nous croyons que, dans la situation actuelle, nous avons un double intérêt à contrôler de très près la fabrication du Duralumin, d'abord pour la construction qui nous occupe, et ensuite et surtout pour nous documenter sur le métal en question qui nous semble devoir jouer dans l'avenir un rôle assez important 
dans certaines catégories de Constructions Mécaniques (moteurs extra-légers appareillage $)^{10}$. »

Ce n'est pas anecdotique car, avec la guerre, l'activité du laboratoire est encore plus cruciale qu'en temps normal, mais il est submergé par des demandes qui émanent de tous les services de l'entreprise, alors que ses moyens humains, très fortement amputés au moment de la mobilisation générale, n'ont pas été reconstitués. L'intérêt de Schneider et Cie se manifeste à un moment où la France, pourtant à l'origine de la production industrielle d'aluminium, prend conscience de son retard dans certaines utilisations de ce métal qui a fait l'objet d'une défiance excessive au cours des années qui ont précédé la guerre. Mais les conditions du conflit facilitent le travail de l'entreprise. En effet, au retour d'une mission de bombardement au-dessus du Royaume-Uni, le Zeppelin L49 s'égare et vient s'échouer le 20 octobre à Bourbonne-lesBains, en Haute-Marne. Cette fois, il est presque intact. L'événement fait grand bruit. Le Zeppelin est exposé aux Invalides et se retrouve même en couverture de l'Excelsior.

Fig.2. Le Zeppelin L 49 abattu à Bourbonne-les-Bains, le 20 octobre 1917

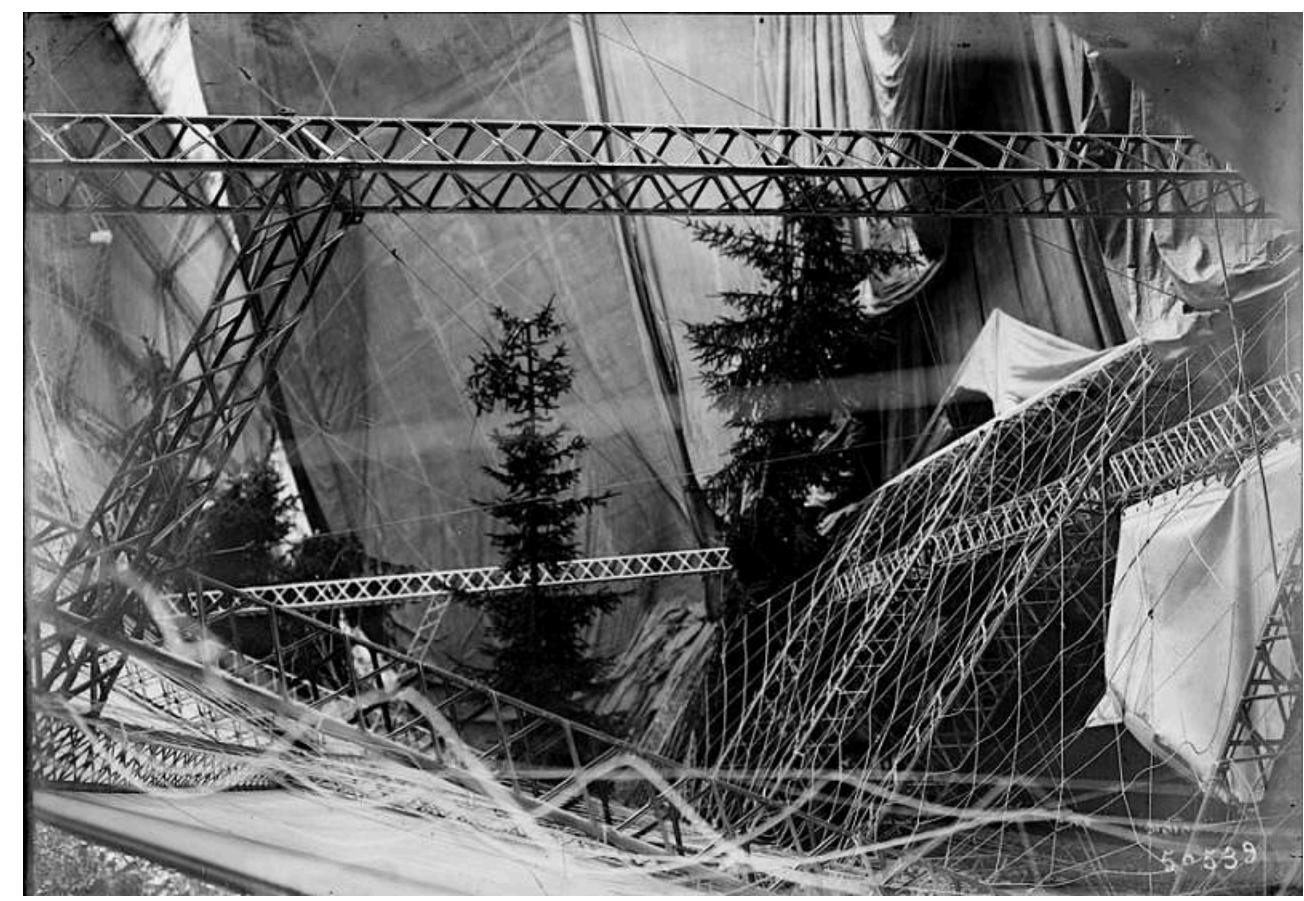

Contrairement à ce qu'indique la légende, ce Zeppelin n'a pas été à proprement parler abattu. À son retour d'une mission de bombardement au-dessus de Londres, il s'était égaré et il échoua, presque intact, à Bourbonne-les-Bains, en Haute-Marne. II put donc faire l'objet d'une inspection minutieuse. Des échantillons furent prélevés à des emplacements précis et envoyés à l'usine du Creusot, afin de connaître la composition de l'alliage avec lequel les éléments de son armature métallique interne avaient été fabriqués.

Photographie de presse, agence Rol, https://gallica.bnf.fr/ark:/12148/btv1b53003814g.item

Le laboratoire du Creusot peut disposer d'un ensemble de pièces métalliques plus complet et en meilleur état ${ }^{11}$. Outre les analyses menées à ce propos, il est possible d'effectuer des comparaisons de certaines pièces par rapport à celles provenant de la Société du Duralumin, en cours d'études aux chantiers de Chalon. Dans l'immédiat, l'étude de la composition des parties métalliques du Zeppelin s'avère d'un intérêt limité, presque rassurant. Leur analyse ne révèle rien que les ingénieurs ne connaissent déjà ou ne sachent pas réaliser. Les mécanismes de rétro-ingénierie popularisés à cette 
occasion n'apparaissent pas. Ils s'avèrent d'une grande déception pour ce qui concerne la logique d'imitation.

Fig.3. Fragment en duralumin de la structure interne du Zeppelin L 49

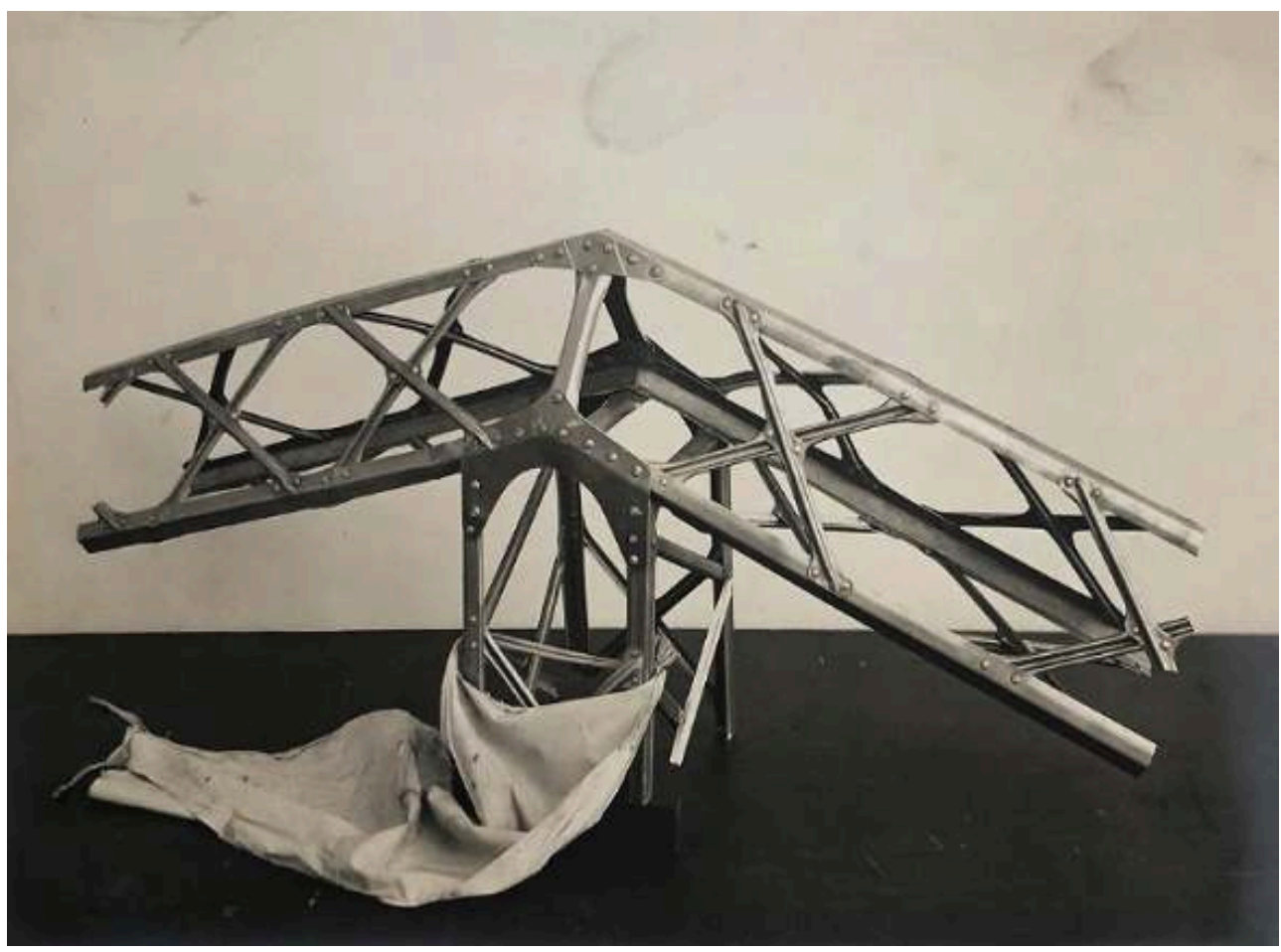

C'est véritablement à partir de ces échantillons reçus en 1917 que le laboratoire du Creusot entame ses premiers travaux concernant l'étude de la composition des alliages légers et de leurs principales caractéristiques. L'entreprise a rapidement perdu le but de ses recherches initiales. II ne s'agit pas de préparer un quelconque retour d'expériences en faveur du soutien à la production d'avions mais bien de répondre à une sollicitation relative à la construction d'un dirigeable de grande dimension, souhaité par la Marine nationale, pour assurer la poursuite des sous-marins allemands dans l'Atlantique.

Archives de l'académie François-Bourdon, Le Creusot

$\mathrm{Au}$ moins est-ce l'occasion de reprendre les études menées de loin en loin sur les alliages légers à base d'aluminium. Entre 1911 et 1914, le laboratoire a été amené à en analyser plusieurs, en particulier du Duralumin et du Silverin ${ }^{12}$. Les différents profilés qui proviennent de l'armature du Zeppelin font l'objet d'essais multiples, de traction, de torsion, d'analyses chimiques et micrographiques, autant d'expériences qui relèvent de la routine quotidienne pour le laboratoire du Creusot. La conclusion des analyses est sans appel: «Il ressort de cet exposé que les alliages soumis à notre examen ne paraissent pas devoir offrir d'intérêt particulier. Leur fabrication paraît peu soignée ${ }^{13}$ ". Les compétences du laboratoire sont suffisamment importantes pour aboutir à des résultats précis, qui vont bien au-delà des attentes de l'expéditeur. L'alliage léger est proche du Duralumin. Les analyses chimiques renseignent l'industriel français sur les difficultés auxquelles l'adversaire est confronté. L'information essentielle ne provient pas de ce qui pourrait être retiré de ses pratiques industrielles. Au contraire, la guerre lui fait perdre compétence et maîtrise technique.

L'alliage récupéré à Revigny est du type " Rübel », enregistré sous le brevet 407814 . Les échantillons sont de composition hétérogène, possèdent des défauts de fabrication et de nombreuses inclusions de contamination, laissant supposer que le choix des 
minéraux de base et fondants se fait sans un contrôle rigoureux de la qualité. Chez l'adversaire, cette question est d'ailleurs l'objet d'âpres critiques de la part du commandement vis-à-vis de certains industriels (Köster 2013). Surtout, l'Allemagne manque de certains composants. Par exemple, la faible teneur en magnésium révèle une carence par rapport aux spécificités connues des alliages légers allemands, confirmant la pression qui s'exerce à l'encontre de certains approvisionnements.

31 À la suite de ces premières recherches engagées en 1916-17, des expérimentations et essais sont poursuivis presque jusqu'à la fin de la guerre. Le département des constructions mécaniques du Creusot entre en action en appui du laboratoire. Il y a donc un intérêt industriel à répondre positivement aux sollicitations dont l'entreprise fait l'objet. Au moment où la question se pose, Le Creusot est contacté non par l'État mais par la Société Anonyme de Navigation Aérienne (SANA). Restée à peu près inconnue, son activité correspond plutôt à celle d'un bureau d'études. Elle cherche à concevoir un dirigeable de grande dimension, à l'imitation des Zeppelin allemands. Audelà de la réalisation sur plan ou à l'état de maquette, il faut un outillage industriel varié, doublé de locaux aux dimensions considérables pour pouvoir mener le projet à son terme.

La SANA recherche donc l'appui contractuel d'une entreprise capable d'engager immédiatement l'étude des éléments métalliques, tout en se plaçant en mesure d'assembler un matériel d'une dimension rarement atteinte, en dehors des vaisseaux de guerre. L'initiative de la SANA découle d'un partenariat avec la Marine pour construire un prototype de croiseur aérien, dénommé F2. Peu d'entreprises allient un même niveau de compétences dans la métallurgie et dans les constructions de structures métalliques. Au sein des établissements Schneider, les ateliers de Chalon-sur-Saône disposent de l'espace nécessaire pour assembler ce dirigeable rigide, d'un volume total de $50000 \mathrm{~m} 3$. Le site regroupe des installations pour la construction de navires de faibles tonnages, torpilleurs et contre-torpilleurs (Passaqui 2007), pour lesquels les commandes sont rares pendant la guerre.

33 Élément plus déterminant, c'est aussi là que Schneider conçoit et prépare les constructions métalliques de grande dimension, comme les gares et les ponts. L'intérêt du projet porté par la SANA incite les établissements Schneider à détacher un de leurs ingénieurs de Chalon auprès d'elle. Car le comportement mécanique des alliages légers, beaucoup plus que la composition chimique, constitue pour Schneider un terrain à défricher, et ceci d'autant plus que l'armature métallique d'un grand dirigeable est composée d'une multitude de pièces qui doivent être extrudées, pliées et rivetées. Mais à défaut de connaître en détail le Duralumin ou les alliages similaires, l'entreprise a pour elle de posséder une grande habitude des épreuves mécaniques des métaux et de disposer déjà de tout l'outillage nécessaire. Dans la campagne d'essais pour mettre au point les alliages légers les plus aptes à répondre aux attentes ainsi que les traitements thermiques qu'ils doivent subir (Cochard 2018), la maittrise acquise de la mise au point des aciers est particulièrement précieuse.

34 À la suite du Creusot, Chalon reçoit des lots d'aluminium de type Duralumin pour les expérimenter avant de les utiliser. Il ne s'agit plus de prises de guerre, mais d'un alliage produit en France, acquis par la SANA. Schneider ne doit pas encore produire l'alliage léger à partir duquel doit être réalisée l'ossature du dirigeable. Son intervention industrielle est sensée se limiter à assembler les pièces métalliques commandées par la SANA à la société du Duralumin, créée en 1912 pour produire sur le sol français cet 
alliage léger de conception allemande ${ }^{14}$. Mais à la même époque, la production de Duralumin en France est en priorité destinée à l'Aviation. Les capacités de production du seul fabricant français apparaissent nettement déficitaires. Elles ne couvrent que $70 \%$ des besoins nationaux, pourtant peu élevés. Le reste doit être importé des ÉtatsUnis dans des conditions de qualité et de régularité qui correspondent mal aux besoins de l'industrie de guerre française.

Avec l'espoir d'une reprise de la fabrication des grands dirigeables en France et les prix élevés que pratique la société du Duralumin du fait de sa rente de monopole, plusieurs acteurs, y compris publics, entendent trouver d'autres interlocuteurs pour répondre à leurs besoins en tôles et profilés, notamment pour la fabrication des longerons. Schneider en profite pour étendre son champ d'action, remonter la filière et se préparer à engager la production d'un alliage léger de sa propre élaboration. Soutenue à partir de 1917 par le ministère de la Guerre, l'entreprise est destinataire du cahier des charges sur lequel s'appuient les relations entre le Service Industriel d'Aviation et la Société du Duralumin. Schneider reçoit alors l'instruction de proposer un alliage possédant les mêmes caractéristiques, ce qui permettrait de diversifier les fournisseurs sur lesquels doit s'appuyer l'industrie aéronautique. L'implication dans la question des alliages légers suscite, dans cette période de conflit, un intérêt croissant.

\section{Début de l'intérêt pour les questions aéronautiques}

Or, en parallèle, Schneider doit redéployer une partie de son activité. Sur le site du Creusot, des espaces sont disponibles depuis l'inauguration de l'usine du Breuil (Passaqui 2019). Certaines fabrications ont été arrêtées, dès lors qu'a été interrompue la production d'obus de petits calibres. L'abandon de la laitonnerie libère l'outillage nécessaire à la production d'alliages légers. En fait, c'est même une aubaine pour l'entreprise qui dispose des moyens pour se procurer, en France ou aux États-Unis, les différents éléments d'addition qui composent l'alliage de Duralumin ${ }^{15}$. Elle envisage dans un premier temps de produire 60 tonnes par mois d'alliage léger à base d'aluminium. Certains des moyens de production sont déjà mobilisables, à la fois pour la fabrication des lingots, mais aussi pour leur laminage.

Les tensions se manifestent dans d'autres services, dans la mesure où l'outillage nécessaire est dispersé sur plusieurs ateliers, tout en étant partagés entre différentes fabrications. Se pose d'ailleurs la question de cet usage commun d'un certain nombre d'équipements. Car si la production et la transformation des alliages légers a profité de machines souvent en excédent, rendues libres par l'abandon de certaines activités, d'autres étaient bien communes. Il serait intéressant de savoir si l'entreprise avait conscience des risques de contamination qu'elle faisait subir à ses produits à base d'aluminium et de leurs conséquences mécaniques et si les problèmes de qualité auxquels elle a été régulièrement confrontée provenaient de la présence conjointe des productions d'acier et d'Alférium ${ }^{16}$.

Le projet de dirigeable tombe dans l'oubli, sans que l'on puisse connaître les raisons, ni même le moment de l'abandon. Comme d'autres recherches, il est complètement resté dans l'ombre. Le temps est au secret, à la dissimulation de certains programmes et l'entreprise a choisi, après 1918, d'éclipser cette page de son histoire. Mais la phase d'études et de réflexion est loin d'avoir été gaspillée. Schneider profite des connaissances accumulées pour reprendre la comparaison de la place respective des 
alliages d'aluminium par rapport aux aciers. Il ne s'agit plus d'en éviter la concurrence mais bien de préparer leur élaboration. Dès le début de 1918, des études sont menées pour étendre l'emploi des alliages légers au sein des différents ateliers des établissements Schneider. L'entreprise se renseigne sur les réalisations menées en ce sens par le concurrent britannique Vickers dans son usine de Birmingham, dont les alliages légers constituent la référence européenne (Hardouin Duparc 2005).

Leur mise en production doit être appréhendée par rapport à leur capacité à devenir des possibles compléments ou alternatives aux aciers spéciaux. Au même moment, Eugène II Schneider détermine la stratégie d'alliances et de concentration qui doit se dessiner au lendemain de la guerre. Il prend conscience que les conditions de production de la fonte et de l'acier risquent de connaître des évolutions sensibles après l'arrêt des combats. L'entreprise envisage déjà de nouer des partenariats avec d'importants industriels européens. Il en découlera une association d'envergure avec le groupe sidérurgique luxembourgeois ARBED (Passaqui 2015). En parallèle et depuis 1915, Schneider et Cie sont engagés dans l'achèvement du vaste complexe industriel de Caen-Mondeville, dans le Calvados (Passaqui 2014).

Les études menées par Louis Bassal, le directeur de l'usine du Creusot, laissent peu d'espoir quant à la possibilité de maintenir, dans le site historique de l'entreprise, la production d'aciers courants. Celle d'acier Thomas est déjà condamnée. Elle a été abandonnée en 1914, dès les premiers jours de la guerre. Mais les projets de diversification géographique de la production d'acier échafaudés à la veille du conflit n'envisageaient pas une extension aussi rapide des capacités de production des aciéries de type Martin en Europe. Avec les nombreuses chutes de métal et les stocks considérables de ferrailles générés par les combats, la production d'acier par ce procédé, déjà en plein essor à la veille de la guerre, risque encore de croître et de concurrencer davantage les produits communs réalisés au Creusot. C'est pourquoi il faut entrer dans une logique similaire à celle retenue quelques années plus tôt par Henri Fayol, à propos du site d'Imphy, dans la Nièvre. Orienté vers les aciers à haute teneur en nickel, puis vers la métallurgie de précision, avec des produits destinés à des usages très spécifiques, il a obtenu un redressement spectaculaire, reposant notamment sur l'excellence de son laboratoire de recherches. Si les quantités écoulées sont faibles, la valeur à la tonne est élevée et les marges bénéficiaires considérables (Duffaut 2000 et 2010). Cependant, se contenter de reproduire au Creusot ce qui a fait le succès de Commentry-Fourchambault et Decazeville à Imphy n'est pas envisageable.

41 Pour approvisionner ses propres ateliers tout en conservant une activité commerciale, Le Creusot doit conserver des capacités de production plus élevées. Pendant que se poursuit la montée en gamme, l'usine doit s'engager dans une production de métal plus diversifiée. Une plus grande proximité est recherchée avec la clientèle. La logique qui prévaut consiste à l'accompagner dans ses choix. Les échanges sont nombreux, précis. Les intermédiaires, en particulier les agences chargées du placement de la production dans un cadre géographique déterminé, s'effacent pour laisser la place à une relation directe, souvent sur une base "peer to peer ». Dans le cas des profilés, des tableaux de comparaisons mettent face à face les qualités des alliages légers par rapport à celles des aciers spéciaux. La légèreté des premiers peut être compensée, à caractéristiques physiques équivalentes, par le volume plus réduit des pièces en aciers spéciaux. Les tôles font l'objet d'une comparaison identique, avec l'idée que l'entreprise pourrait élaborer, de concert, les deux gammes de produits métallurgiques. 

productions de guerre. Au cours de cette période, les applications industrielles ont été nulles. Les travaux sont restés confinés au sein du laboratoire. C'est d'ailleurs là qu'ils reprennent en 1921, toujours à l'instigation des services de l'Aviation. Il est vraisemblable que les résultats engrangés pendant la guerre ont été incomplets. La deuxième impulsion ouvre rapidement la voie vers des applications industrielles d'autant plus souhaitées que l'entreprise pâtit de la disparition des commandes militaires que les constructions civiles sont loin de compenser. Le site du Creusot est en surcapacité et, avec lui, certaines de ses dépendances et filiales. Schneider doit chercher à se confronter à de nouvelles fabrications, au fur et à mesure qu'elles émergent. Il en découle une évidente dispersion et, souvent, une incapacité à peser sur les nouveaux marchés autrement qu'en imposant ses produits, dans le cadre de partenariats capitalistiques temporaires.

Dans ces conditions, la demande de la part des armées de reprendre la mise au point d'un alliage léger encourage l'entreprise à constituer en son sein, une activité nouvelle qui, dans la tradition de ce qu'elle mène depuis son origine, partirait toujours de 
l'élaboration du métal pour aller jusqu'à la réalisation de l'objet technique complet et utilisable. Dans un premier temps, la reprise de l'expérimentation et de la mise au point d'un nouvel alliage s'opère toujours dans le périmètre du laboratoire, mais l'avancée du projet conduit, en novembre 1922, à la création d'une section "alliages légers ", chargée de mener l'achèvement des travaux de recherche avant la phase de fabrication. Elle est intégrée au sein de la Direction des Mines et de la Métallurgie (DMM). Ses orientations stratégiques sont déterminées à Paris, par la Direction de l'Exploitation. Son activité au Creusot est répartie au sein des services du forgeage et des laminoirs. Les produits longs relèvent de l'usine de Bordeaux. La première concrétisation de l'entrée dans les alliages légers découle du dépôt, le 4 mai 1923, de l'alliage commercialisé sous la marque Alférium ${ }^{20}$.

Évolution de la composition de l'alliage léger développé au Creusot entre 1921 et 1922

\begin{tabular}{|l|l|}
\hline Alliage léger Schneider en 1921 & Alliage léger Schneider en 1922 \\
\hline $\mathrm{Cu} 3 \%, \mathrm{Zn} 2 \%, \mathrm{Mn}$ 0,5\%, Mg 0,5\%, Si 0,5\% & $\mathrm{Cu} 3,25 \%, \mathrm{Mn}$ 0,5\%, Mg 0,5-0,6\%, Mn 0,5\%, Si 0,5\% \\
\hline
\end{tabular}

Ses caractéristiques sont similaires à celles du Duralumin. Le Creusot a d'ailleurs multiplié les expériences à partir de différents lots de cet alliage au cours des mois qui précèdent la mise au point de l'Alférium ${ }^{21}$.

Fig.4. Plaquette de présentation des produits des usines Schneider

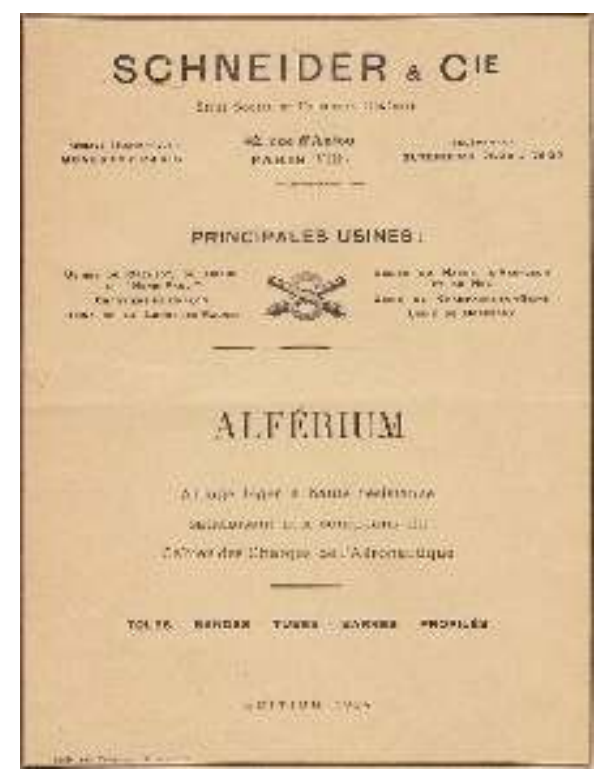

Les établissements Schneider déposent leur propre marque correspondant à un alliage léger dès 1923. Bien que les commandes militaires soient rares, à cette époque, dans le domaine des avions de guerre, l'entreprise s'engage dans les constructions aéronautiques. Par contre, alors que cette activité devait permettre de garantir une partie de l'activité des différentes usines de l'entreprise, c'est en fait le site historique qui profite de la naissance de la section « alliages légers ».

Archives de l'académie François-Bourdon, Le Creusot

Au cours des années suivantes, l'entreprise oriente cette nouvelle fabrication vers trois axes, celui de l'obtention de tôles et bandes notamment pour l'aviation, mais aussi pour d'autres activités. En effet, ces produits reçoivent ultérieurement d'autres usages. Par 
exemple, dans les années Trente, ils constituent la base de la carrosserie des autorails de type Micheline. Leur conception particulière implique d'utiliser des alliages légers pour réduire la charge sur les essieux ${ }^{22}$. Le Creusot remporte d'importants contrats, en raison de son savoir-faire dans les équipements destinés à l'aviation, Michelin empruntant beaucoup techniquement à ce domaine pour abaisser la masse de son autorail. Schneider et Cie se lancent surtout dans la conception d'avions très innovants pour lesquels le métal se subsisterait intégralement au bois et à la toile. Déjà, avant la fin de la guerre, l'entreprise avait commencé à tracer les contours d'un avion d'assaut, peu adapté d'ailleurs à la mission à laquelle il était dévolu. L'évaluation de ses caractéristiques avait été faite quelques jours après la fin du conflit, ce qui avait conduit, dans les semaines suivantes, à l'abandon du projet, peu après l'interruption des recherches dans le domaine des alliages légers (Favre 2002).

Contrairement aux aspects relatifs à la mise au point d'un alliage équivalent au Duralumin, les programmes aériens des établissements Schneider sont bien connus. Ils ont fait l'objet d'un article étoffé rédigé par Georges Bondoux, à partir des archives de l'Académie François Bourdon, au Creusot (Bondoux 2007), précédé d'une étude richement illustrée reposant sur les fonds du musée de l'Air et de l'espace du Bourget (Liron, Borget, Bénichou 2000). De cette volonté de l'entreprise d'associer la production d'avions à son volumineux catalogue de fabrication découlent plusieurs initiatives capitalistiques. Parmi celles-ci se distingue la Participation Henri-Paul, Jean Schneider ${ }^{23}$, dont le capital est appelé par tranches successives en 1920. Administré par Jean Schneider, le fils cadet d'Eugène II Schneider, son but est précisé dans les statuts :

«Les parties ci-dessus désignées, désireuses de contribuer au développement de l'aviation et d'aider dans un but de défense nationale à la réalisation d'un programme de fabrication intensive, plus particulièrement en ce qui concerne les avions puissants, de tir et de bombardement, ont décidé d'unir leurs efforts et de former entre elles une association en participation définie et réglée par les dispositions du présent acte. Article 1er. Il est formé entre les soussignés une association en participation ayant pour but : l'étude de toute question se rattachant à la construction et à l'armement d'avions, plus particulièrement d'avions et d'hydravions de tir et de bombardement ${ }^{24}$."

50 L'article $\mathrm{V}$ reprend un des aspects les plus courants des pratiques commerciales de l'entreprise, en réservant à Schneider et Cie la fourniture de tous les matériaux et de l'outillage qui devraient équiper les ateliers de cette société. Car il ne s'agit pas seulement de produire des alliages légers, mais bien, comme dans le cas de l'acier, d'en être le principal consommateur. Il n'est pas possible d'implanter au Creusot même des ateliers d'assemblage d'avions. Le manque de place dont souffrent déjà les différents ateliers limite l'intervention de l'usine historique aux activités d'élaboration de l'alliage et de forgeage qui l'accompagnent. Par contre, avec l'arrêt presque complet des productions d'artillerie, certains sites Schneider peinent à conserver l'élan de la guerre et, parmi eux, l'ensemble situé à proximité du Havre.

51 C'est pourquoi la décision est prise d'y assembler le premier appareil conçu par les établissements Schneider. Quand l'entreprise s'intéresse à la conception et à la construction d'avions de grande dimension, c'est à nouveau dans ce site que 
l'assemblage et les essais de résistance trouvent des halles de dimension suffisante ${ }^{25}$. Car le premier modèle correspond à un avion imposant, destiné au bombardement, un quadrimoteur biplan muni de moteurs Lorraine-Dietrich. Dans la continuité de toutes les nouveautés de l'entreprise au lendemain de la guerre, ce modèle est baptisé HenriPaul ${ }^{26}$. Au moment du salon aéronautique de Paris, en 1922, l'avion Schneider est présenté à côté de certains des futurs grands noms de la construction aéronautique, comme Latécoère ou Morane-Saulnier.

Par rapport aux solutions techniques de la période, le prototype amplifie et même systématise le rôle du métal en lieu et place de la toile et du bois. Il en découle un appareil d'une masse élevée, plus de dix tonnes, pour lequel les conditions de portance doivent faire l'objet d'une attention soutenue. Les solutions techniques sont retenues sur la base d'expériences menées en soufflerie, auprès du laboratoire Eiffel notamment, à partir d'une maquette au $1 / 30^{\text {e }}$. Elle a d'ailleurs servi à d'autres étapes de la conception de l'appareil, et en particulier pour l'établissement de la position de son centre de gravité.

53 Cet avion occupe une place particulière dans les programmes aériens contemporains. Son design est soigné mais ses dimensions le rendent peu aptes aux missions pour lesquelles il a été conçu. Par contre, à la même époque, les bombardiers de nuit sont déjà présents dans le ciel. Certains ont montré leurs capacités à aller bien au-delà des lignes ennemies pour remplir leur mission, en réduisant les capacités de concentration des hommes et des matériels par le bombardement, par exemple, des nœuds ferroviaires. La Grande-Bretagne a obtenu un succès majeur en déployant les bombardiers Handley Page type 0 , notamment le $0 / 400$. Opérationnel à partir de 1918, il possède des qualités qui le font apparaître comme une alternative plus que crédible aux dirigeables de type Zeppelin. Ainsi, les recherches entreprises par Schneider en vue de produire un croiseur aérien ont pris une tournure qui n'avait pas été soupçonnée à l'origine, en s'appuyant sur les études réalisées dans le domaine des alliages légers. Du dirigeable, Schneider passe à l'avion de bombardement.

L'entreprise met en avant les deux grandes qualités de sa réalisation, une homogénéité et une résistance qui sont difficiles à obtenir en utilisant une structure en bois. Avec l'Alférium, elle participe au mouvement favorable à l'utilisation des alliages légers à base d'aluminium, dont les qualités mécaniques connaissent une succession de progrès. Mais elle s'appuie aussi sur les travaux qu'elle mène depuis près de trente ans sur les aciers spéciaux pour lesquels certaines pièces restent nécessaires pour former l'armature des appareils aériens.

La maîtrise technique des alliés au nickel et au chrome ayant été obtenue dans le cadre de ses travaux sur les blindages et les canons, il s'agit désormais d'établir une complémentarité, en les utilisant en association avec les alliages légers. Certaines entreprises aéronautiques, comme Lorraine-Dietrich, sont à la fois clientes pour les alliages légers et les aciers spéciaux. En fait, le seul point pour lequel les établissements Schneider ne parviennent à aller au bout de leur démarche se situe au niveau des ailes, qui restent entoilées, à un moment où le laminage des tôles en duralumin ou alliages légers équivalents reste difficile à maîtriser.

En 1922, le bombardier Henri-Paul fait l'objet d'une publication promotionnelle au sein de la revue Le Génie civil. 
Fig.5. Coupe du quadrimoteur Henri-Paul, assemblé et mis au point dans les ateliers Schneider et Cie du Havre

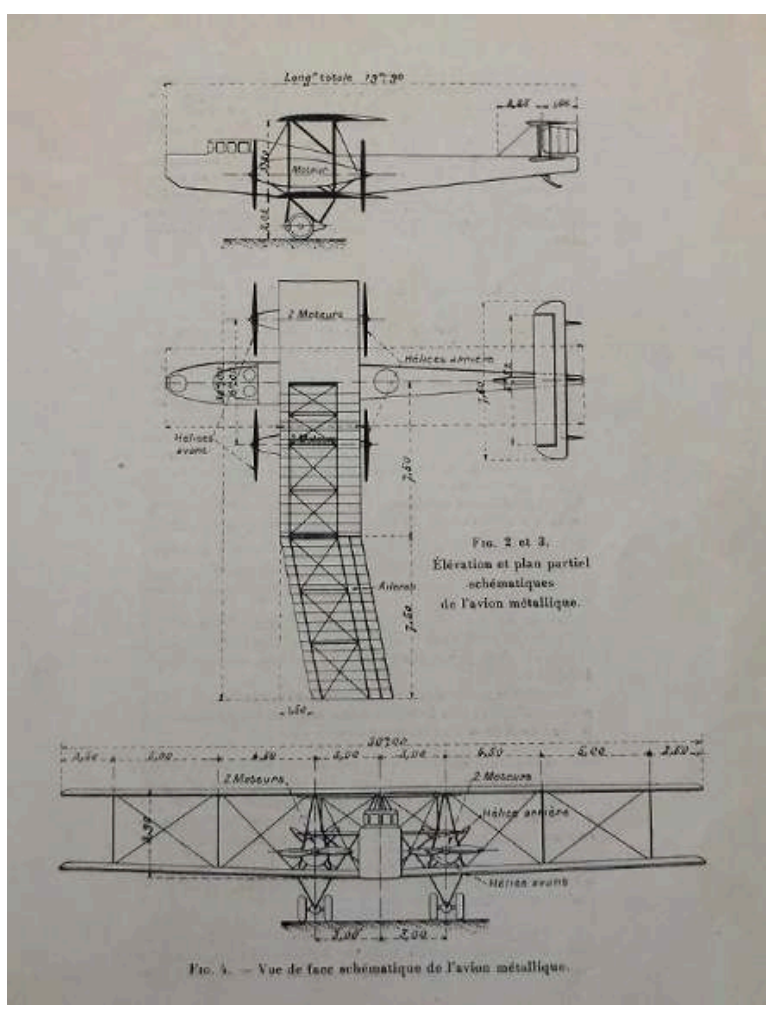

Élévation et plan partiel schématiques de l'avion, accompagnés d'une vue de face de l'avion. Le plan partiel met notamment l'accent sur la structure interne métallique d'une des ailes de l'appareil.

Le Génie civil, 2 décembre 1922. Archives de l'académie François Bourdon, Le Creusot.

Fig.6. Mise au point de l'armature métallique de l'avion Henri-Paul dans la Grande halle des usines du Havre

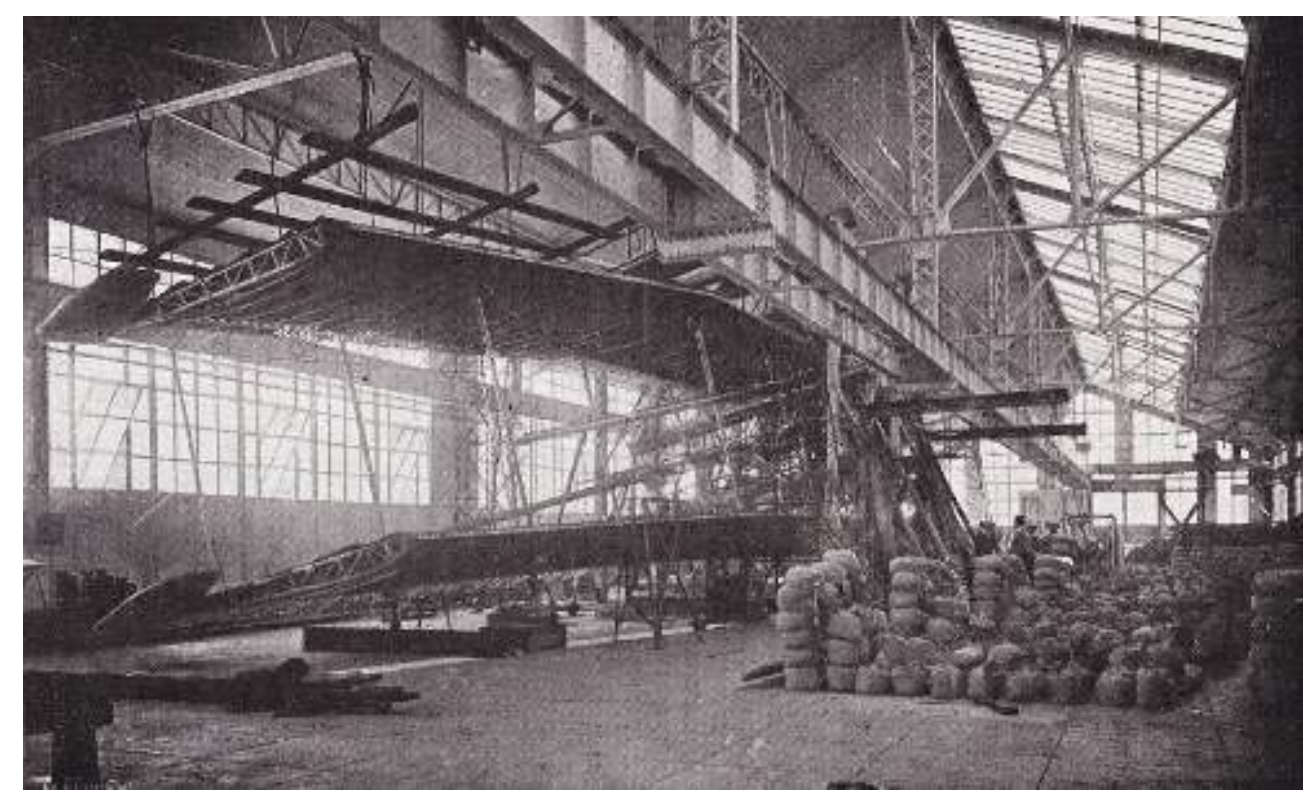

Cette photographie permet notamment de se rendre compte de la complexité du montage de l'ossature des ailes et de la carcasse de l'appareil. L'ensemble de l'attirail qui enserre l'appareil est destiné à la phase d'essai statique de la cellule.

Le Génie civil, 2 décembre 1922. Archives de l'académie François Bourdon, Le Creusot 


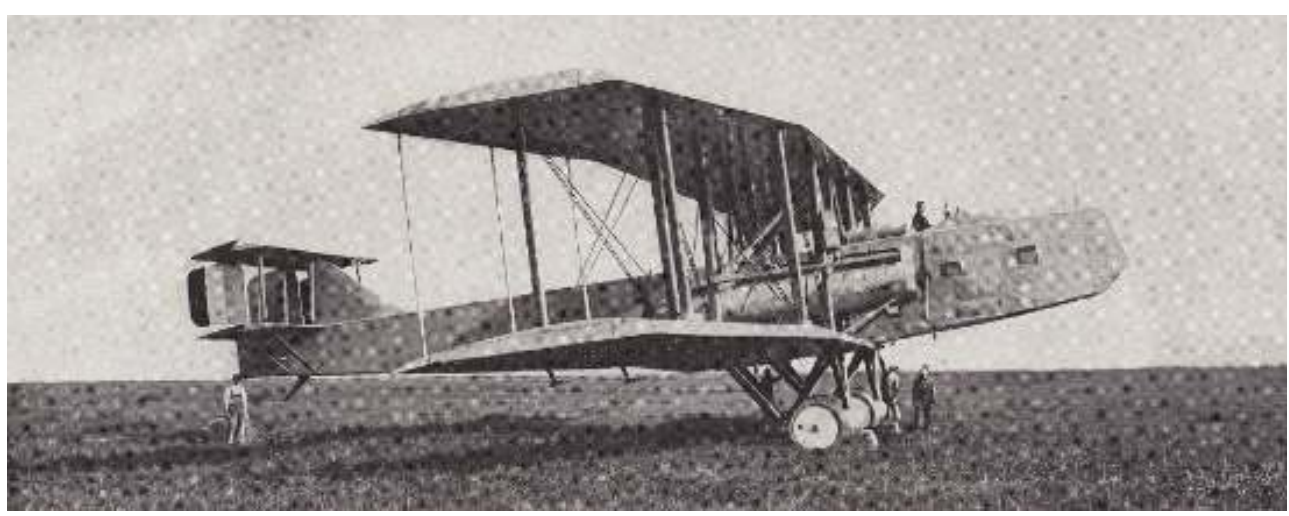

Vue de profil au cours d'une campagne d'essais sur le terrain d'Harfleur, aménagé à proximité des usines Schneider du Havre.

Le Génie civil, 2 décembre 1922. Archives de l'académie François Bourdon, Le Creusot.

L'appareil entre en phase de test à l'aérodrome de Villacoublay, qu'il a d'ailleurs rejoint par ses propres moyens. Mais la campagne d'essais en vol révèle que l'avion est rigide, difficilement maniable. Il reste à l'état de prototype. Il n'en fournit pas moins une maîtrise technique mobilisée pour d'autres modèles, notamment le Schneider 10M. Ce monoplan de chasse, bimoteur, muni d'une double dérive, à nouveau en grande partie métallique, permet à l'entreprise de faire connaître ses travaux sur l'Alférium et, ce faisant, d'asseoir sa réputation dans le domaine des alliages légers. Mais l'appareil est à nouveau confronté à certaines des difficultés rencontrées sur le prototype Henri-Paul. Bien que d'autres modèles soient annoncés, l'affaire reste sans suite ${ }^{27}$.

Le risque est important, à la fois d'échec et d'entacher la réputation des établissements Schneider. Par ailleurs Eugène II est soucieux de se défaire de l'image de marchand de canons à laquelle il est associé pendant les années 1920. Or, la production de bombardiers pourrait relancer les polémiques politiques auxquelles il est déjà confronté. En outre, depuis 1913, l'entreprise a pris l'habitude de créer des filiales au moment de s'engager dans de nouvelles branches industrielles, tout en maintenant avec elles des relations de dépendance très fortes (d'Angio 2000). La création d'une filiale spécifique, AVIMETA, (Société pour la Construction d'Avions Métalliques), société au capital de 2 millions de francs, répond à cette superposition d'intérêts et d'orientations stratégiques. Mais, ce qui est mis en avant dans sa présentation, ce sont les compétences dans l'aviation métallique :

« Il est universellement admis que l'avion commercial de l'avenir sera entièrement métallique. Les avantages de ce type de constructions sont multiples: insensibilité aux intempéries, indéformabilité, conservations des qualités de vol, incombustibilité, longue durée d'amortissement, interchangeabilité des éléments, etc. Par contre, ce procédé exige des études longues et difficiles appuyées sur de nombreux essais de laboratoires et une longue pratique d'atelier, si l'on veut réduire en même temps le poids de l'avion et son prix de revient, tout en augmentant l'homogénéité et la sécurité de la construction. Seule en France, la Société pour la construction d'avions métalliques «AVIMETA » possède à l'heure actuelle une 
expérience de la fabrication d'avions entièrement métalliques, lui permettant de lutter avec succès contre la concurrence allemande. Fondée avec l'appui de MM. Schneider et Cie qui lui ont fait apport de leur documentation, de leurs installations et de leur personnel technique, elle bénéficie de leurs importantes études entreprises depuis 1920. La société "AVIMETA» est parvenue à un stade suffisamment avancé dans le perfectionnement de sa technique pour pouvoir normaliser, non seulement ses procédés de construction, mais aussi des éléments et même des ensembles interchangeables... » 28

59 Ses installations correspondent en fait à celles d'un bureau d'études qui n'est jamais allé au-delà de la présentation de prototypes ou, au mieux, d'exemplaires de pré-séries. Dans le prolongement des modèles Schneider, elle s'engage en premier lieu dans la conception d'avions militaires. AVIMETA n'entend ne pas circonscrire son activité aux appareils de grande envergure. Faute de rencontrer le succès, elle propose aussi des chasseurs avant d'envisager la mise au point d'appareils à usage civil, qui débouche sur la présentation du trimoteur AVM $132^{29}$. Les avions d'AVIMETA sont reconnus pour la qualité de leur ligne. Ils sont esthétiques, aérodynamiques et proposent souvent des solutions qui seront reprises par la suite. Pour plus de $60 \%$ de leur masse, ils sont composés d'Alférium. Il est d'ailleurs stipulé par contrat que le fuselage doit être en alliage léger Schneider. Mais la filiale aéronautique des établissements Schneider fait, d'un point de vue industriel et commercial, du surplace.

En fait, ses appareils reposent sur un design qui n'évolue pas et reproduit les mêmes contraintes que celles découvertes depuis les premiers vols du modèle Henri-Paul. Le choix des matériaux aboutit toujours à des appareils lourds et peu maniables. Échec commercial, succès technique très mitigé, l'aventure AVIMETA s'achève en 1929 par le désengagement des établissements Schneider à la suite du rejet par les donneurs d'ordre de l'AVIMETA 121 et l'abandon de toute ambition dans la production complète d'avions (Bondoux 2007). La fin de l'expérience ne soulève pas de traumatismes. AVIMETA disposait bien à Courbevoie d'un grand hall pour assurer l'assemblage des appareils, mais l'essentiel du travail se faisait manuellement, sans pratiquement aucun recours à de l'outillage.

61 La question du maintien partiel des ambitions dans le domaine aéronautique demeure d'actualité cependant, car il faut préserver l'importance industrielle de certains sites. Il est primordial en effet de renouveler le domaine de compétences d'usines de circonstance. C'est notamment le cas de la douillerie de Bordeaux. Entrée en service en juin 1915, elle a été en mesure de produire 10 millions de douilles. Avant même l'arrêt des hostilités, sa production est réorientée vers des produits destinées à assurer sa pérennité, alliages légers ou à base de cuivre entre autres, et en particulier des profilés destinés à l'aviation ${ }^{30}$. A défaut de devenir un acteur de premier plan dans le domaine aéronautique, Schneider propose ses compétences aux constructeurs établis. L'entreprise se lance alors dans la sous-traitance, en particulier pour les héliciers. 


\section{Schneider, un sous-traitant au service de l'industrie aéronautique}

$62 \mathrm{Au}$ cours de ce bref épisode consacré à la conception d'avions, Schneider entre en relation avec certains de ses futurs clients pour un emploi ciblé de l'Alférium. Des démarchages sont opérés auprès des premiers fabricants d'hélices métalliques, notamment les établissements Levasseur (Liron, Borget, Bénichou 2000). La production des ébauches d'hélices débute, au Creusot, en $1924^{31}$. Elle découle d'une mission confiée aux États-Unis à un des ingénieurs du site. À la suite de contacts pris sur place, Le Creusot constitue une des premières étapes du voyage en Europe de l'inventeur américain Reed ${ }^{32}$. L'usine profite de sa renommée internationale. Mais les prétentions financières de Reed paraissent exagérées pour une activité incertaine. C'est donc au producteur d'hélices Levasseur qu'il cède le droit d'utilisation de son brevet NoUS1836989 (Lévy 2009). Les relations techniques et commerciales entre Levasseur et Schneider qui débutent alors $^{33}$, vont au-delà de la seule livraison des ébauches. Schneider soutient le producteur d'hélices dans sa volonté de mettre au point ou d'acclimater en France d'autres alliages légers ${ }^{34}$. C'est pourquoi, en 1924, Le Creusot tente d'imiter le Skleron, un alliage aluminium-lithium réputé pour être particulièrement résistant ${ }^{35}$.

Composition et comparaison du Duralumin et du Skleron, alliages d'aluminium

\begin{tabular}{|l|l|l|}
\hline Type d'alliage & Composition & Caractéristiques \\
\hline Duralumin & $2,5-5,5 \% \mathrm{Cu}, 0,5-2 \% \mathrm{Mg}, 0,5-1 \% \mathrm{Mn}, 0,2-1 \% \mathrm{Si}$ & Très solide \\
\hline Skleron & $12 \% \mathrm{Zn}, 3 \% \mathrm{Cu}, 0,5 \% \mathrm{Mn}$, traces de Fe, Si, Li & Très dur \\
\hline
\end{tabular}

Source : https://doc.rero.ch/record/323166/files/2018_BA-CRAE-Despland_Chlo_Identification_Alliages_Aluminium_et_caract_risation_produits_corrosion.pdf

63 La marque Alférium doit s'imposer par rapport aux autres alliages légers, en particulier face au Duralumin. Au bout de quelques années, le marché des alliages pour les hélices se structure autour de binômages. Chaque constructeur établit une relation privilégiée avec un métallurgiste. Outre le tandem Schneider et Levasseur, Le Duralumin est proche de Breguet, et Ratier de Gnome et Rhône. Levasseur est un partenaire exigeant, confronté à un marché concurrentiel (Lévy 2009), qui entend être soutenu par son partenaire commercial, au niveau des questions de prix, mais aussi pour supporter une partie des efforts de recherches en cours : «Faisant état des sacrifices journellement consentis par sa maison pour répandre l'hélice métallique, M. Fréchet ${ }^{36}$ estime que nous devons l'aider et que les essais à entreprendre vis-à-vis de la réalisation d'ébauches matricées rentrent dans le cadre de la contribution que nous devons apporter à l'effort commun continu qui est à faire pour nous maintenir au premier rang des fabricants d'hélices en métal léger ${ }^{37}$. » 
Fig.8. Chantier de fusion et de coulée de l'Alférium, coulée d'un lingot pour hélice au Creusot.

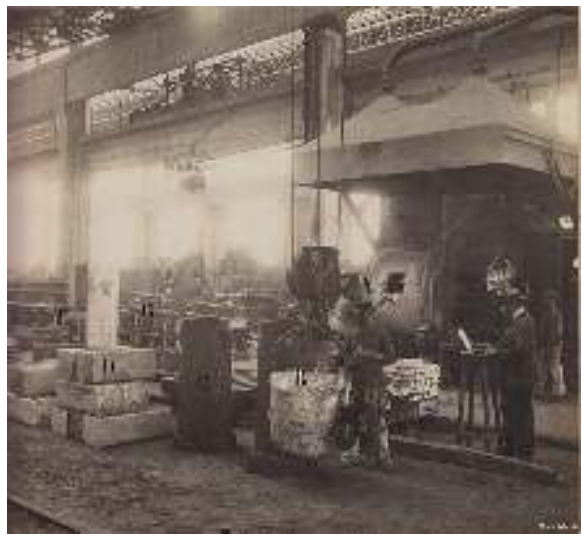

Cette première photo d'une série de quatre prises de vue date de 1926. Elle indique les principales étapes et caractéristiques de l'outillage. A. Chargement de petits lingots d'aluminium, dans un four électrique à arc, type Detroit. B. Prise de la température en poche du métal venant d'être coulé. C. Lingotières à lingots pour Hélice. E. Lingotières à plaques. F. Plaques pour tôles et bandes. G. Lingotières à billettes. $\mathrm{H}$. billettes.

Archives de l'académie François Bourdon, Le Creusot

Fig.9. Forge du Creusot. Presse hydraulique Schneider.

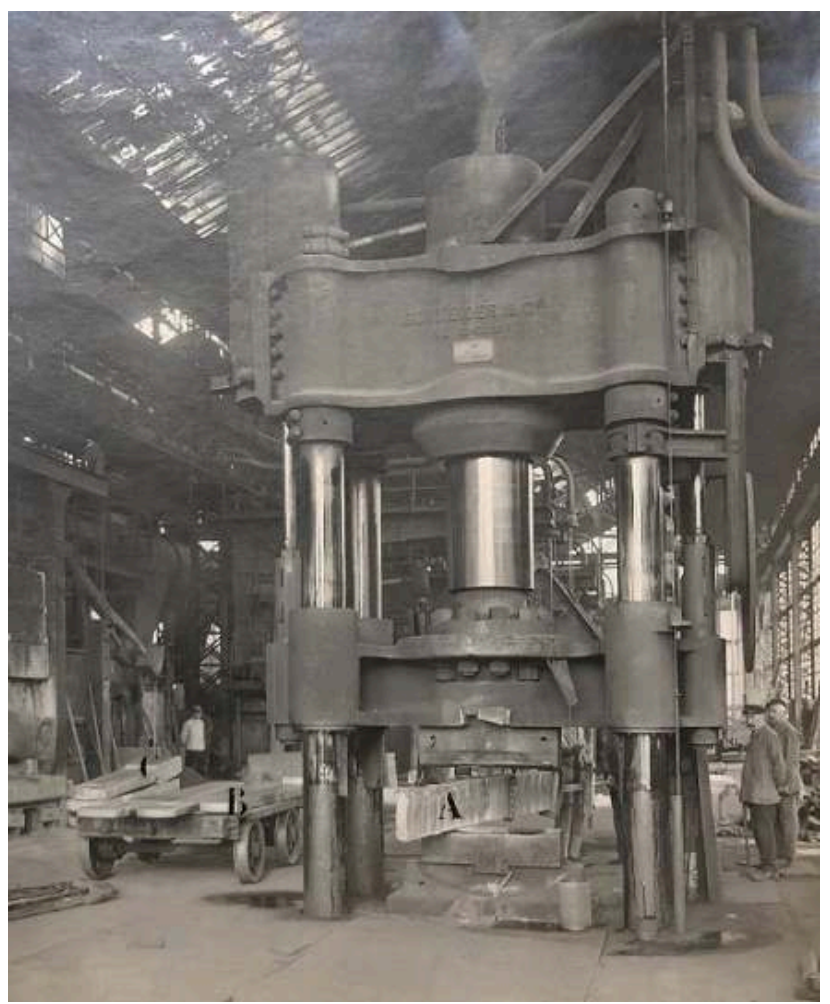

Bloomage d'un lingot d'Alférium pour obtenir une ébauche pour hélice (transformation d'un bloom en ébauche). A. Bloom en finissage sous la presse de 1500 tonnes. B. Blooms terminés. C. Blooms avant forgeage final.

Archives de l'académie François Bourdon, Le Creusot 
Fig.10. Forge du Creusot. Petit marteau pilon.

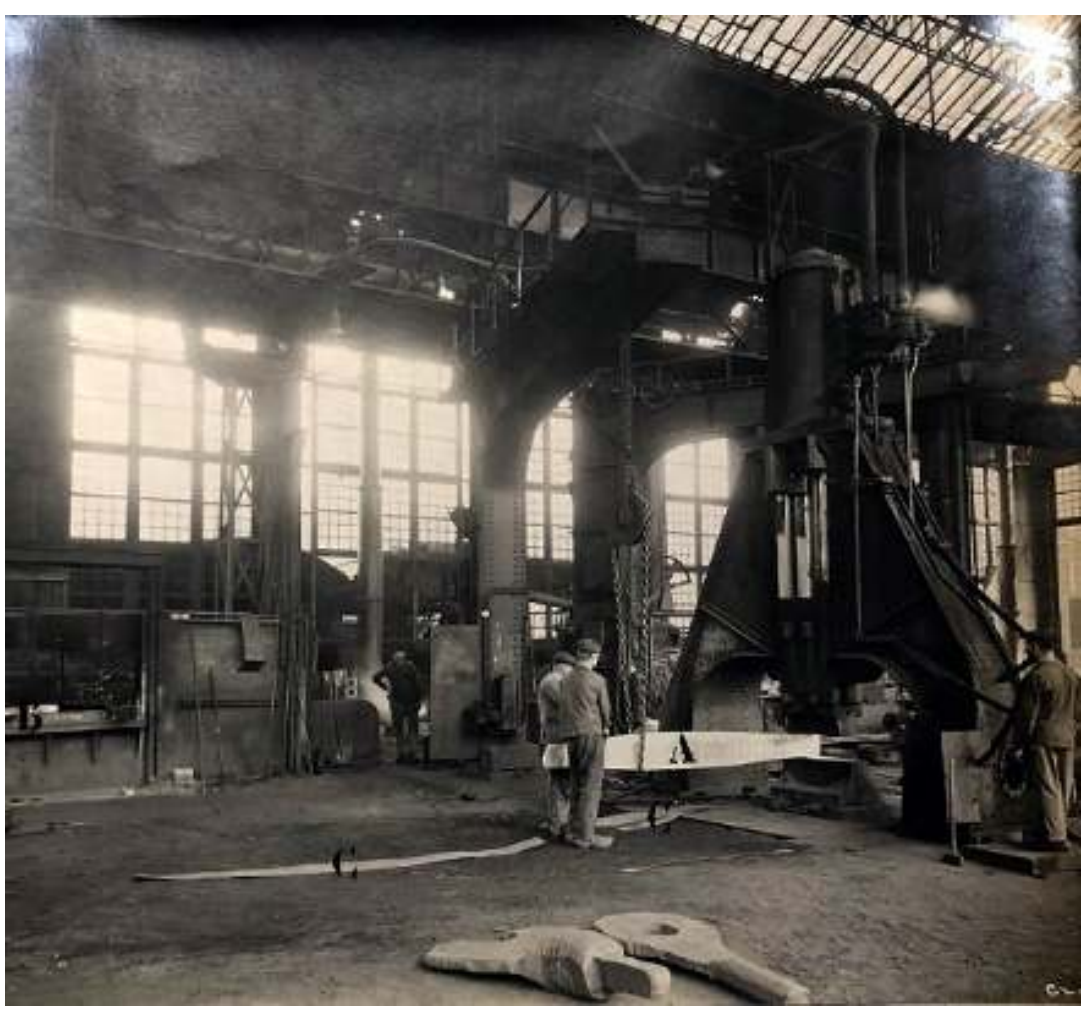

Fabrication des ébauches pour hélices. Amincissement des pales destinées au fabricant d'hélices Levasseur. A. Ébauches en finissage au pilon de 3 tonnes. B. Morceaux de bloom débité, en chauffage. C. Calibre de vérification des produits en cours de forgeage.

Archives de l'académie François Bourdon, Le Creusot. 
Fig.11. Usine du Creusot. Première tentative de fabrication d'une ébauche d'hélice monobloc pour les établissements Levasseur

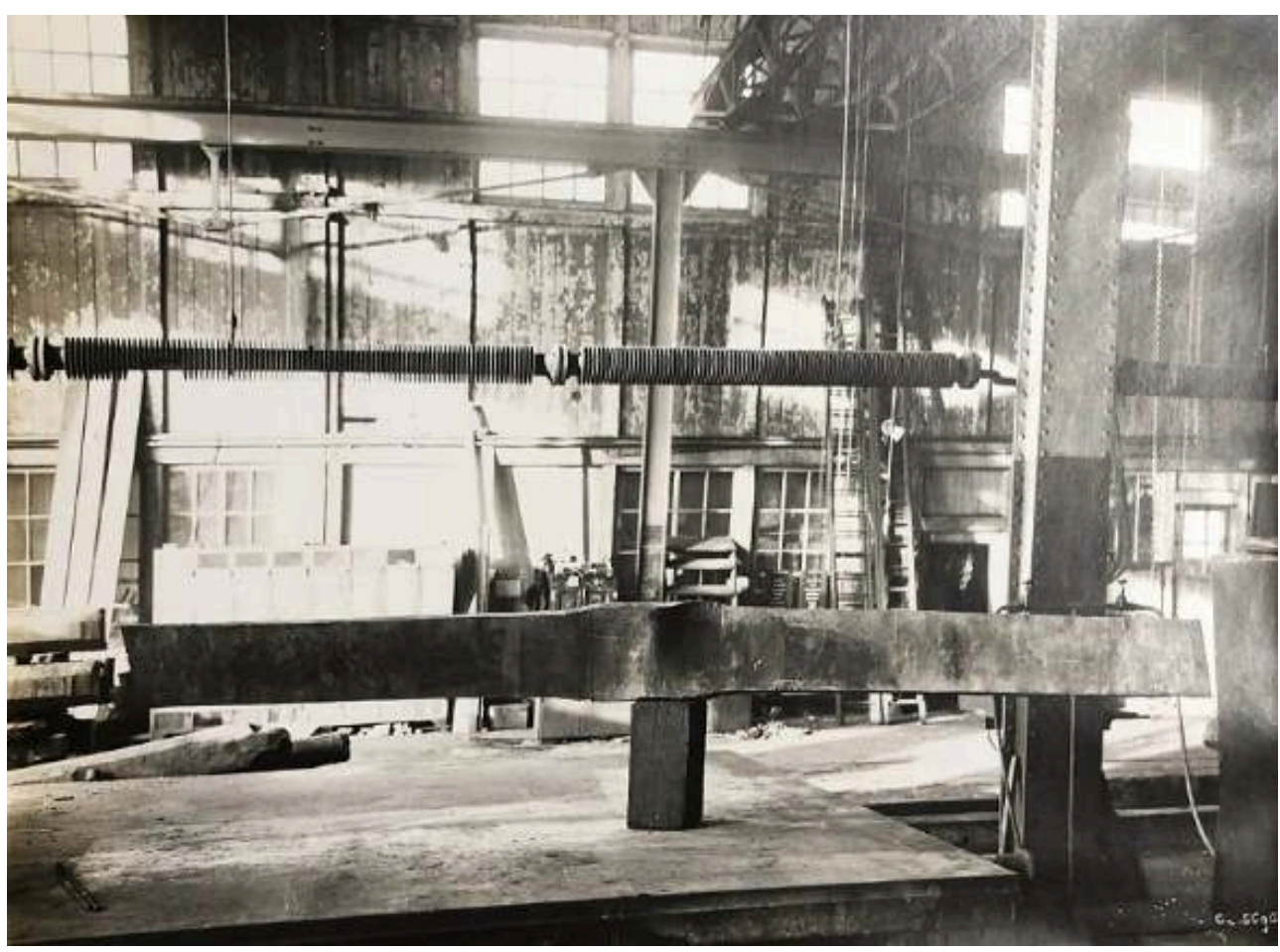

Les premières tentatives de fabrication d'ébauches d'hélices à moyeu monoblocs sont réalisées à l'automne 1926. La mise au point des conditions de forgeage a impliqué une réflexion complexe autour des conditions d'utilisation des presses hydrauliques.

Archives de l'académie François Bourdon, Le Creusot. connaître précisément l'outillage de Levasseur, ainsi que l'orientation de l'entreprise. Les ébauches fournies par Schneider se rapprochent de plus en plus du produit fini, ce qui implique pour le métallurgiste de supporter une grande partie du coût de l'adaptation de l'outillage aux orientations techniques de Levasseur, pour que cette dernière n'ait plus qu'à réaliser une partie de plus en plus réduite de l'usinage. Mais avec une telle logique, Schneider ne peut se satisfaire du partenariat que s'il porte sur des commandes d'ébauches en grandes séries et non sur une multitude de modèles d'hélices, comme les deux entreprises avaient l'habitude de pratiquer, au moment de l'amorce de leurs relations techniques et commerciales.

Le déplacement d'une partie de l'activité du fabricant d'hélices vers le métallurgiste est en fait révélateur de la vulnérabilité financière de Levasseur qui, après avoir engagé d'importants investissements et fait un réel effort pour suivre les progrès enregistrés dans le domaine de la fabrication d'hélices aux États-Unis et en Allemagne, n'a plus les moyens d'entretenir l'amélioration de son parc de fours et de machines qui finit par être frappé d'obsolescence. Pour Schneider, cette situation n'est pas inquiétante au niveau industriel car l'entreprise a les moyens d'étendre son champ de compétences. Elle prend conscience de la fragilité de son partenaire et en subit les conséquences par l'absence de régularité dans les commandes. Schneider doit produire sans disposer d'un programme de fabrication bien établi, avec l'impression que Levasseur cherche à réduire à ses dépens les encours de production de manière à ne pas trop altérer sa trésorerie ${ }^{38}$. 

Peu de fournisseurs français peuvent répondre aux caractéristiques des fours recherchés. Ce sont le plus souvent des équipements acquis aux États-Unis ou produits sous licence en France. Le recours au four électrique à arc n'est pas nouveau au Creusot. Son utilisation avait débuté pendant la guerre pour obtenir certaines nuances d'acier. La maîtrise technique avait dans un premier temps été aléatoire, faute de disposer d'un personnel formé. Ce sont ces équipements qui sont repris par le service des Alliages légers. Mais, quand, au milieu de 1922, les commandes de lingots d'Alférium s'envolent $\mathrm{du}$ fait de la demande aéronautique, Le Creusot installe un four type Detroit avec lequel il gagne en capacité, chaque coulée étant plus importante que l'ensemble des coulées quotidiennes obtenues antérieurement ${ }^{40}$. Le choix s'est porté vers cet équipement dans la mesure où il permettait d'obtenir un alliage plus homogène. C'est un préalable indispensable en vue de résoudre une partie des critiques qui se portent sur le comportement de l'Alférium.

Outre les problématiques engendrées par le laminage se pose aussi celle de l'irrégularité des lingots reçus par les clients. Pour diminuer les prix de revient, les lits de fusion reposent sur un apport en aluminium sous deux formes. Les bains sont composés à parité de lingots et de riblons, c'est-à-dire de déchets qui, pour la plupart, proviennent de l'entreprise elle-même. Leur emploi est indispensable pour assurer le recyclage des chutes. À un moment où les prix des intrants sont volatiles, l'entreprise 
peut réduire les oscillations de ses prix de revient. Dans le cas de la production d'acier, les riblons constituent une matière première de choix. Ils représentent la base des coulées d'acier. Mais pour l'élaboration de l'Alférium, leur utilisation dans de fortes proportions dégrade la qualité et suscite des remontrances régulières des clients. En accord avec les capacités de mesure du laboratoire, il faut repartir de zéro, c'est-à-dire se passer complètement des riblons pour n'utiliser que de l'aluminium très pur acquis sur le marché.

70 À partir de ce point de départ, le service des Alliages légers reprend et augmente progressivement l'incorporation des chutes. Ces additions successives servent à déterminer le point d'équilibre entre la maîtrise des coûts et le respect du cahier des charges imposé par le client. Les campagnes d'essais permettent d'obtenir cet équilibre à partir d'une incorporation de déchets réduite à 25-30\%. C'est trop peu pour garantir le maintien de la rentabilité du nouveau service et pour écouler le stock de riblons. Mais si le site du Creusot est reconnu pour l'excellence de ses ingénieurs métallurgistes, la décision de conserver une partie importante des productions des établissements Schneider découle aussi de la maîtrise technique de ses ouvriers. Sur le site du Creusot, la contraction du personnel a été sévère, mais une production diversifiée a pu être maintenue, entretenant la place essentielle de l'usine au sein des établissements Schneider et Cie.

71 Par son histoire, sa maîtrise technique, la population ouvrière du Creusot a peu d'équivalent en France. C'est ce qui explique pourquoi Schneider et Cie, au contraire de certains de leurs concurrents, ont souhaité maintenir l'essentiel de leurs activités métallurgiques au Creusot. Avec l'accumulation des compétences, les progrès constatés sont suffisamment importants pour améliorer la compétitivité du nouveau service. Entre 1922 et 1925, les évolutions sont lentes, mais elles sont suffisantes pour garantir la présence de la section "Alliages légers", sur la base des conditions édictées au moment de sa création. Quand une enquête interne est engagée en 1926 à propos du maintien du service des alliages légers au sein de l'entreprise, la réponse est positive, mais un arbitrage doit intervenir entre Le Creusot et l'usine de Bordeaux, où elle aurait dû être localisée et où sont déjà produits les tubes et les profilés en Alférium ${ }^{41}$.

La réponse est sans appel, et correspond bien au haut degré de technicité des alliages légers : « la direction de l'exploitation est opposée à la fabrication éventuelle du métal Alférium à l'Usine de Bordeaux, alternative qu'avait envisagée la Conférence du 1er juillet 1926. La métallurgie des alliages légers doit rester à l'Usine du Creusot qui l'a mise au point. Elle possède seule des cadres rompus aux études métallurgiques et disposant des moyens d'études appropriés à cette technique très spéciale. On ne peut même pas envisager la fabrication de l'Alférium à Bordeaux après lancement de la fabrication par Le Creusot, car le métal Alférium n'est vraisemblablement qu'un premier terme dans la série des alliages légers industriels destinés à évoluer dans les mêmes conditions que les aciers spéciaux. L'élaboration des alliages légers doit donc rester du ressort d'une usine métallurgique. $»^{42}$ Car la maîtrise du procédé de la fabrication reste à améliorer.

Un plus grand soin est accordé à l'acquisition de l'aluminium. Il est accompagné d'une remise en lingot préalable des riblons, qui ne sont plus incorporés dans le bain de métal sous forme de déchets. Le retour à une parité entre l'aluminium acheté et celui recyclé est obtenu en avril 1923. L'environnement de travail autour du four électrique est modifié de manière à faciliter les manutentions et la mise en lingot. Avec une 
production plus stable, l'ébauchage et l'usinage de grandes pièces d'Alférium peuvent reprendre dans des conditions économiques et techniques satisfaisantes ${ }^{43}$. Cette répétition d'essais, de tâtonnements, de retours en arrière pour revenir à la solution initiale est symptomatique des caractéristiques de la phase d'apprentissage. Pour l'acier, l'entreprise possède un savoir-faire rare en Europe, dans la mise au point de nuances qui correspondent à des usages bien déterminés. Par contre, elle ne dispose que d'un seul alliage léger, qui ne convient pas à tous les besoins de la clientèle.

74 Pour éviter de perdre une partie de ses débouchés, l'entreprise utilise une autre de ses forces. En s'appuyant sur sa capacité à couler une gamme étendue de produits métallurgiques qui sont obtenus à partir de brèves campagnes dédiées à une nuance particulière, Le Creusot entretient son aptitude à répondre sur-le-champ à des commandes très différentes. La même logique est adaptée à la fabrication de l'Alférium. Pour les tôles d'avions qui constituent le produit le plus délicat à mettre au point et pour lequel les récriminations de la clientèle sont les plus nombreuses, l'entreprise accepte un surcoût de son prix de revient en limitant l'introduction de riblons ${ }^{44}$. Dans le cas des autres débouchés, elle conserve le principe de l'équilibre entre lingots d'aluminium et déchets relingotés.

La dissociation des coulées en fonction de l'usage final permet d'atteindre plus régulièrement un degré élevé de satisfaction du client, ce qui n'empêche pas de rester attentif aux plaintes. Après plusieurs années de balbutiements, la réussite de l'industrialisation de l'Alférium repose sur l'association d'innovations qui s'additionnent aux routines de l'entreprise. Elles émergent au sein d'un environnement productif nouveau et foisonnant. Comme les lingots et pièces métalliques adressés à la clientèle sont utilisés dans des conditions bien spécifiques, les échanges avec elle sont constants. Lorsque des défauts sont signalés, ils sont identifiés pour trouver une solution rapide. Des ingénieurs de l'usine du Creusot sont plus particulièrement chargés de répondre aux attentes des utilisateurs. Ils se rendent régulièrement dans les ateliers qui travaillent à partir de l'Alférium, afin de conseiller mais aussi de prendre en compte les éventuelles critiques. Ils constatent qu'elles proviennent parfois d'utilisateurs qui maîtrisent mal les conditions de stockage ou d'emploi.

Mais quand le défaut provient du service des alliage légers, sa faible importance et la relative liberté dont il dispose lui permettent de réagir avec vivacité. Il ne peut se contenter des positions commerciales acquises. La concurrence s'affirme dans le domaine des alliages légers. Elle est le fait de petites entreprises, agressives, dont la structure industrielle permet une adaptation plus rapide que celle du grand complexe. C'est pourquoi, au Creusot, la question des alliages légers est traitée à part. Les routines productives sont récentes et imparfaites. Des modes opératoires qui semblaient maitrisés peuvent encore connaître des pertes de qualité qui menacent les relations avec des clients nouveaux et prometteurs.

C'est ce qui se produit avec l'entreprise italienne Ansaldo, confrontée à des défauts graves de certaines pièces. La réaction est radicale, dès lors qu'une inspection des commandes incriminées révèle que le problème technique est interne. Schneider et Cie analysent l'ensemble des conditions de production. Les procédures sont éclairantes par ce qu'elles disent des moyens adoptés en vue ne pas perdre la confiance du client. Il faut déjà le rassurer en montrant que l'on met en place en sa faveur des moyens de remédiation importants, presque ostensibles. Tous les aspects du problème sont 
envisagés pour essayer d'en comprendre l'origine, en l'occurrence le manque de compacité des pièces ${ }^{45}$.

Devant l'étonnement que suscite l'ampleur des défauts, l'atelier en charge des essais amorce le raisonnement suivant : essayer toutes les solutions possibles pour éliminer l'une après l'autre celles qui ne sont pas la source des incidents. Progressivement, l'origine des défauts parvient à être identifiée. Il survient au moment de la solidification du métal, ce qui fait dire aux ingénieurs métallurgistes : « le problème est cette fois bien posét6 ${ }^{\prime}$. À la suite de plusieurs campagnes consacrées à l'observation des conditions de coulées, l'atelier parvient à une amélioration sensible qui permet de conclure: "Nous ne croyons pas que ces principes de coulée soient appliqués dans aucune autre fonderie d'alliages légers. Nous pensons que les résultats obtenus avec notre pratique actuelle nous mettent en très bonne posture vis-à-vis de nos concurrents ${ }^{47}$. »

79 Parmi les principaux aspects de ce processus d'amélioration, l'entreprise peut s'appuyer sur une organisation productive aboutie. Les difficultés soulevées font l'objet d'une réflexion qui n'implique pas un service, mais repose bien sur une mise en relation des compétences. Les différents ateliers s'épaulent d'un point de vue technique, mais aussi commercial au moment d'établir les conditions de production en fonction des spécifications du client. Par rapport aux conditions du marché, l'usine du Creusot peut pousser plus ou moins loin la fabrication. Le fait qu'il s'agisse d'un secteur nouveau, dans une période d'incertitude économique, se traduit par de nombreuses défaillances.

Le Creusot peut être amené à suppléer la disparition de ses partenaires vitaux. Lorsque le fabricant d'hélices Levasseur disparaît en $1935^{48}$, Le Creusot tente de reprendre à son compte la fourniture de pales d'hélices en métal. Il entre en contact avec les principaux clients de l'entreprise en cours de disparition et se voit immédiatement proposer des marchés auprès des grands noms de l'aviation, comme Hispano-Suiza. Cette entreprise reçoit les ébauches adressées par Schneider pour leur donner la forme voulue, avant de les renvoyer à l'entreprise sidérurgique qui s'occupe de la trempe. Les pièces retournent enfin chez Hispano qui termine l'usinage. Schneider et Hispano se sont partagé l'intervalle laissé vacant par la disparition de Levasseur. Hispano en est venue, comme le faisait déjà Gnome-et-Rhône, à conduire conjointement la production de moteurs et des éléments propulseurs, les hélices en l'occurrence. Son partenariat industriel avec une maison aussi renommée que Schneider l'incite à engager de grands investissements, à un moment, le début des années 1930, où la France découvre le besoin de renouveler et augmenter sa flotte d'avions de guerre ${ }^{49}$.

\section{Conclusion}

81 Ainsi, au cours des années qui précèdent la Seconde Guerre mondiale et malgré la disparition de son partenaire privilégié, Schneider est resté un acteur de poids dans la production d'alliages légers. Pour certains produits, comme les tôles et les bandes, les problèmes de fabrication se sont avérés structurels, notamment au niveau du planage. L'entreprise a eu du mal à s'imposer. Elle connaît d'ailleurs les raisons des difficultés qui lui sont propres. Malgré la qualité de sa main-d'œuvre et de son outillage, elle souffre de leur absence de spécialisation, par rapport aux entreprises concurrentes qui inscrivent leur activité industrielle dans un périmètre plus restreint $t^{50}$. Les rencontres avec les partenaires commerciaux, comme Nieuport, sont un moyen de mettre en 
exergue les défauts des fabrications du Creusot, puisque les tôles de l'usine sont stockées à côté de celles de concurrents et, ne serait-ce que visuellement, elles apparaissent sales et souffrent de la comparaison.

Il n'en demeure pas moins que dans le cadre de la production d'ébauches d'hélices, elle a pu renouveler sa présence en cessant d'être dépendante d'un seul fabricant. Au contraire, elle a multiplié les relations avec les constructeurs aéronautiques ${ }^{51}$. Elle satisfait aussi à leurs besoins en fournissant des galettes d'Alférium utilisées pour certaines pièces des moteurs d'avions ${ }^{52}$. L'ensemble ne représente qu'une faible contribution aux activités de l'usine du Creusot et, par extension, de celles des établissements Schneider. Mais dans le domaine industriel qui correspond à ses compétences initiales, les résultats ne sont pas négligeables. Fidèle à sa culture, elle a avancé avec prudence, de façon à apprendre et comprendre progressivement le comportement de son alliage léger, pour pouvoir réagir lorsque de nouvelles perspectives commerciales se présentaient ${ }^{53}$.

À partir de 1922, c'est quand, à nouveau, l'entreprise est allée au-delà des seules activités métallurgiques pour s'engager dans le champ des constructions mécaniques qu'elle s'est retrouvée en difficulté. Après des débuts hésitants, voire chaotiques pendant et au lendemain de la Première Guerre mondiale, les établissements Schneider et Cie décidèrent de reprendre les études relatives aux alliages légers, sans esprit d'abandon. L'expérience acquises en l'espace de quelques années, les amena à considérer que cette activité, implantée au Creusot et à Bordeaux, était entrée dans une phase de maturité. Néanmoins, ce fut sans trouver la rentabilité, du fait de l'instabilité de l'environnement économique, mais aussi des multiples tâtonnements qu'il fallut effectuer avant de proposer une marque répondant aux besoins de la clientèle aéronautique ${ }^{54}$.

Après 1926, le volume des commandes devint suffisamment significatif pour que l'entreprise décide de faire le bilan des résultats enregistrés. Pour la première fois, elle prépare un véritable plan organisant par paliers les investissements à entreprendre. Ce pan de l'histoire des établissements Schneider est resté peu connu. En fait, si l'entreprise est régulièrement citée comme partenaire des constructeurs aéronautiques pendant l'entre-deux-guerres, la production et la transformation d'alliages légers ne représente qu'une fraction infime de l'activité de l'ensemble de l'entreprise. Le site du Creusot n'était pas prévu, initialement, pour constituer la base industrielle d'une activité qui aurait dû remplir les carnets de commandes des usines de l'entreprise menacés par la fin des hostilités. Pourtant, l'usine historique des établissements Schneider demeura la plus impliquée et la plus performante pour cette question des alliages légers.

ALLOITTEAU Jean, «Les Zeppelins attaquent à Revigny ", Matériaux pour l'histoire de notre temps, 2, 1985, p.32-35

D'ANGIO Agnès, Schneider et Cie et la naissance de l'ingénierie : des pratiques internes aux responsabilités extérieures, 1836-1949, Paris, CNRS éditions, 2000

BEAUD Claude, «L'innovation dans les établissements Schneider (1837-1960) », Histoire, économie et société, 1995, $4^{\mathrm{e}}$ trimestre, p.501-518

BERGERON Louis, Le Creusot, une ville industrielle, un patrimoine glorieux, Paris, Belin, 2001

BERGERON Louis, «La maison Schneider dans l'avant-guerre et dans la Première Guerre mondiale : un témoignage inédit ", Autour de l'industrie, histoire et patrimoine. Mélanges 
offerts à Denis Woronoff, Paris, Comité pour l'histoire économique et financière de la France, 2004, p. 419-420

BONDOUX Georges, "Schneider et Cie, constructeur aéronautique ", Bulletin de l'Académie François Bourdon, t.VIII, 2007, p.22-28

CARON François, Les deux révolutions industrielles du XX ${ }^{e}$ siècle, Paris, Albin Michel, 1997

CARON François, La dynamique de l'innovation, changement technique et changement social, (XVI ${ }^{e}$-XXe siècle), Paris, NRF Gallimard, 2010

CHADEAU Emmanuel, De Blériot à Dassault, histoire de l'industrie aéronautique en France, 1900-1950, Paris, Fayard, 1987

CHEZEAU Nicole, De la forge au laboratoire, naissance de la métallurgie physique (1860-1914), Rennes, Presses Universitaires de Rennes, 2004

CHEZEAU Nicole, « Les traitements thermiques de l'acier : progrès des connaissances et des applications (1900-1940) ", in L'acier en France : produits et marchés, de la fin du XVIII siècle à nos jours, Dijon, EUD, 2006, p.21-38

COCHARD Audrey, Microstructures et propriétés mécaniques des alliages de type Duralumin du Breguet 765 n०504 64-PH : approche historique et science des matériaux, thèse de doctorat, Matériaux, Université de Toulouse 3 Paul-Sabatier, 2016

DUFFAUT François, «La recherche à Imphy entre 1895 et 1914 », Actes du colloque La situation du Nivernais en 1900, $5^{\mathrm{e}}$ rencontres d'histoire de la métallurgie, Les Amis du Vieux Guérigny, 2000, p.97-102

DUFFAUT François, «Imphy, innover pour vivre ", La Nièvre, le royaume des forges, Études et documents $n^{\circ} 8$, Nevers, CG58, 2006, p.37-43

DUFFAUT François, «Pierre Chevenard (1888-1960)», Marteau Pilon, t.XXII, 2010, p. 65-66

FACON Patrick, DEBAECKER Jean-Pierre, Ballons et dirigeables, Proxima éditions, 2001

FAVRE Julien, Histoire des établissements Schneider au Havre, Mémoire de maitrise d'histoire contemporaine, sous la direction de Jean-Pierre Chaline, Paris IV Sorbonne, 2002

FONTANON Claudine, «La naissance de l'aérodynamique expérimentale et ses applications à l'aviation: une nouvelle configuration socio-technique », Histoire de la mécanique appliquée: enseignement, recherche et pratiques mécaniciennes en France après 1880, Cahiers d'histoire et de philosophie des sciences, n46, 1998, p.62-75

(1780-1880. Les non-ferreux et l'industrialisation, Rennes, Presses Universitaires de Rennes, 1998

GARÇON Anne-Françoise, "Inventer le Bessemer et perdre l'acier: histoire d'une disruption cognitive (1855-1875) », Entreprises et histoire, n 98 , 2020, p.78-93 GRAMPAIX, Jean, L'hélice, son histoire, ses techniques, Éditions d'Argenson, 1966

GUIMINOT Élodie, TISSIER Yolaine, «Étude des alliages d'aluminium pour la mise au point des traitements de conservation des avions ", Cahiers d'histoire de l'aluminium, $\mathrm{n}^{\circ} 54,2015, \mathrm{p} .3-13$

108 HARDOUIN DUPARC Olivier, "Alfred Wilm et les débuts du Duralumin », Cahiers d'histoire de l'aluminium, n³4, 2005, p.63-77 
KÖSTER Roman, «Zeppelin, Carl Berg, and the Develpment of Aluminium Alloys for German Aviation (1890-1930)», Cahiers d'Histoire de l'aluminium, vol.1, n50, 2013, p. 72-87

LEVY Geoffroy, Invention et développement de l'hélice aérienne, 1840-1930, Mémoire de master 2 sous la direction d'A-F. Garçon, Université Paris 1 Panthéon-Sorbonne, 2009 LIRON J., BORGET M., BENICHOU M., « Quand le groupe Schneider faisait de l'aviation de guerre ", Fana de l'aviation, $n^{\circ} 363$, février 2000

112 MIOCHE Philippe \& WORONOFF Denis (dir.), L'acier en France, produits et marchés, de la fin du XVIII siècle à nos jours, Dijon, Éditions Universitaires Dijonnaises, 2006

PASSAQUI Jean-Philippe, La stratégie des Schneider, du marché à la firme intégrée, 1836-1914, Rennes, Presses Universitaires de Rennes, collection Histoire, 2006

114 PASSAQUI Jean-Philippe, "Schneider et Cie, de la sidérurgie aux constructions navales ", Actes du Colloque Marines et entreprises, XVII ${ }^{e}$-XIXe siècles, septièmes rencontres d'histoire de la métallurgie, 2007, p.235-256

115 PASSAQUI Jean-Philippe, « De l'acier aux aciers spéciaux, des aciers spéciaux aux aciers à haute teneur en nickel », Marteau Pilon, t.XXIV, juillet 2012, p.23-34

PASSAQUI Jean-Philippe, « Eugène II Schneider et la sidérurgie lorraine, au lendemain de la Première Guerre mondiale ", Bulletin de l'Académie François Bourdon, $\mathrm{n}^{\circ} 16$, avril 2015, p.22-28

PASSAQUI Jean-Philippe, "Produire le char Schneider CA1-75S, une aventure industrielle des établissements Schneider et Cie », Bulletin de l'académie François Bourdon, t. XIX, 2018, p.10-19

PASSAQUI Jean-Philippe, « Mobilisation des facteurs de production et coordination de l'activité industrielle aux usines Schneider du Creusot de 1914 à 1918 ", in Patrick FRIDENSON et Pascal GRISET (dir.), L'industrie dans la Grande guerre, Paris, Comité pour l'histoire économique et financière de la France, 2018, p.299-324 PASSAQUI Jean-Philippe, "La Première Guerre mondiale: un tournant décisif dans l'histoire de la sidérurgie française », Bulletin de l'Académie François Bourdon, t.XX, 2019, p.18-25

PRUDHOMME Arnaud, Pierre Levasseur et ses avions, Paris, TMA éditions, 2002

\section{NOTES}

1. Ce texte a bénéficié d'informations complémentaires et de conseils apportés par Jean Desbiens, de l'Université Laval (Canada) et François Duffaut, ancien directeur de la R\&D des aciéries d'Imphy. Qu'ils soient remerciés pour leur sollicitude et pour la qualité de leurs propositions.

2. Académie François Bourdon (AFB), Salon Schneider, 1130-05, Établissements Schneider pendant la guerre, effort industriel, aciéries, transformation et marche des aciéries pendant la guerre, Le Creusot, le 3 septembre 1919. 
3. Floris Osmond et Jean Werth, «Théorie cellulaire des propriétés de l'acier », Annales des Mines, 1885, p.25-84.

4. Base du four formée de matériaux réfractaires.

5. AFB, 01G0704, Société du Duralumin, duralumin, métal inoxydable, sd. Le slogan de la société est d'ailleurs : « Légèreté de l'aluminium, Résistance de l'acier ».

6. Qui correspondent aujourd'hui à l'alliage d'aluminium ASTM 2024 (Cochard 2018).

7. AFB, Salon Schneider, 1130-05, Effort de guerre pendant la période 1914-1918, Forgeage, Le Creusot, 2 septembre 1919.

8. AFB, Salon Schneider, Moteur d'aviation « Lorraine ", Notice descriptive, conduite et entretien, Paris, Schneider et Cie, sd.

9. AFB, 01G0152-04, Ateliers de constructions, essais effectués sur divers fragments d'alliage d'aluminium provenant de la carcasse du Zeppelin abattu à Revigny, le 10 juillet 1916.

10. AFB, 01G0584, Note du département des Constructions Mécaniques pour Usine du Creusot, Paris, le $1^{\mathrm{er}}$ septembre 1917.

11. AFB, 01G0166-43, Usine du Creusot, laboratoire, essais effectués sur quelques éléments du Zeppelin L49 tombé à Bourbonne-les-Bains, Le Creusot, $1^{\text {er }}$ décembre 1917.

12. AFB, 01G0704, Essais CM (pour Constructions mécaniques), métal «Silverin» - Bronze «Gélior », mars 1914.

13. AFB, 01G0166-43, Usine du Creusot, laboratoire, essais effectués sur quelques éléments du Zeppelin L49 tombé à Bourbonne-les-Bains, Le Creusot, $1^{\text {er }}$ décembre 1917.

14. AFB, 01G0584, Métal léger pour aviation et dirigeables, cahiers des charges, Paris, le 7 décembre 1917.

15. AFB, 01G0584, SANA, Note sur la production de Duralumin, Paris, le 3 septembre 1917.

16. Réflexion suggérée par Jean Desbiens, dont l'importance n'apparaît pourtant pas dans la documentation très étoffée conservée à l'Académie François Bourdon.

17. En raison de la faible résilience de l'aluminium au froid, et ceci comparativement à l'acier (Information transmise par Jean Desbiens). Pour autant, actuellement, des chantiers de constructions navales français, produisent, à l'instar d'OCEA, des bateaux à usage maritime, de type patrouilleurs.

18. AFB, 01G0584, Aéroplanes Breguet, Courrier à M. Coqueugnot, Vélizy, le 5 septembre 1917.

19. AFB, 01G0584, Usine du Creusot, Laboratoire, Voyage à Paris, maison Breguet, les 4 et 5 juin 1918, Le Creusot, 10 juin 1918.

20. AFB, Salon Schneider 0420-07, Alférium, historique de sa fabrication aux usines du Creusot, section alliages légers, usines du Creusot, novembre 1926.

21. Ibid.

22. AFB, Usine du Creusot, service laminoirs, compte rendu de la visite de M. Lenoble aux établissements Rozé, à Levallois-Perret, le 8 juillet 1936.

23. AFB, 187 AQ 538 - c - 13, Participation Henri-Paul. Il s'agit de la raison sociale. Le capital est détenu par Jean Schneider, son père Eugène II et les établissements Schneider.

24. AFB, 187 AQ 538 - c - 13, Participation Henri-Paul.

25. "Schneider et Cie, usines du Havre, d'Harfleur et du Hoc", L'Illustration économique et financière, Seine-Inférieure, sd. p. 6.

26. En souvenir d'Henri-Paul Schneider, fils aîné d'Eugène II Schneider, mort pour la France en combat aérien en 1918. Ce prénom est aussi porté par la fonderie et la cokerie métallurgique construites au lendemain de la guerre à Montchanin, près du Creusot, ainsi que par un nouveau puits de mine, à la houillère de La Machine, dans la Nièvre.

27. "L'avion métallique quadrimoteur type Henri-Paul des établissements Schneider ", Le Génie Civil, tiré à part, 1922, $20 \mathrm{p}$.

28. AFB, 30K0001-01, AVIMETA, avion commercial trimoteur, type AVM 132, Courbevoie, août 1927 (AVM pour AVIMETA). 
29. $\mathrm{AFB}, 30 \mathrm{~K} 0001-01$, Ministère du Commerce et de l'Industrie, aéronautique et transport aérien, marché $\mathrm{N}^{\circ} 253 / 7$ en date du 13 juin 1927, marché de gré à gré pour la fourniture d'un avion de transport public.

30. «Schneider et Cie, usine de Bordeaux », L'Illustration économique et financière, la Gironde, sd. p. 1.

31. AFB, Salon Schneider 0420-07, Alférium, historique de sa fabrication aux usines du Creusot, section alliages légers, usines du Creusot, novembre 1926.

32. Ibidem.

33. Ibid.

34. Ibid.

35. Ibid.

36. Directeur des établissements Levasseur.

37. AFB, 01G0084-A-931, Rochette, Usine du Creusot, forgeage, mission à Paris et Düsseldorf, 22 au 30 octobre 1929.

38. AFB, 01 G0085-B-1025, Usine du Creusot, forgeage, compte-rendu d'une visite de MM. Faucillon et Jacqueson aux établissements Levasseur le 4 avril 1930.

39. AFB, Salon Schneider 0420-07, Alférium, historique de sa fabrication aux usines du Creusot, section alliages légers, usines du Creusot, novembre 1926.

40. Ibidem.

41. AFB, 01G0077-B-628, Usine du Creusot, Mission de M. Faucillon à l'usine de Bordeaux, les 9 et 10 août 1926.

42. AFB, 01G0077-A-626, Usine du Creusot, Mission de M. Faucillon à Paris, du 25 au 29 juillet 1926.

43. AFB, Salon Schneider 0420-07, Alférium, historique de sa fabrication aux usines du Creusot, section alliages légers, usines du Creusot, novembre 1926.

44. Ibidem.

45. Lié à l'espacement des structures cristallines atomiques.

46. AFB, 01 GO 159-03, Dechaume, Fonderies Henri-Paul, Alférium, sur les défauts internes des lingots d'Alférium, causes et remèdes, septembre 1927.

47. Ibidem.

48. https://www.hydroretro.net/etudegh/heliciersfrancais.pdf

49. AFB, 01G0095-B-1872, Usine du Creusot, Forgeage, Mission de M. Rochette, à Paris, le 4 février 1936.

50. AFB, Salon Schneider 0420-07, Alférium, historique de sa fabrication aux usines du Creusot, section alliages légers, usines du Creusot, novembre 1926.

51. AFB, 01G0095-B-1872, Mission de M. Rochette à Paris, Hispano-Suiza, Breguet, Gnome et Rhône, 31 janvier-1 ${ }^{\text {er }}$ févier 1936.

52. Pour parvenir à bonnes fins, Le Creusot s'appuie sur les informations en provenance de Gnome-Rhône, à partir de leurs propres commandes auprès des aciéries de Firminy, qui, elles, emploient du duralumin. AFB, 0064Z3741-02, Usine du Creusot, Note du Département des Mines et de la Métallurgie, Le Creusot, 12 novembre 1927.

53. AFB, 0064Z3741-02, Usine du Creusot, Note du Département des Mines et de la Métallurgie, Le Creusot, 22 octobre 1927.

54. AFB, 01G0077-A-626, Usine du Creusot, Mission de M. Faucillon à Paris, du 25 au 29 juillet 1926. 


\section{RÉSUMÉS}

Pendant la Première Guerre mondiale, les établissements Schneider et Cie sont confrontés à plusieurs sollicitations qui les conduisent à s'intéresser à la question des alliages légers à base d'aluminium. Forts de leurs compétences dans la mise au point d'aciers spéciaux, grâce notamment à la présence, au Creusot, d'un laboratoire métallurgique d'excellence, l'entreprise parvient à déposer sa propre marque en 1923 : l'Alférium. En parallèle, elle décide d'appliquer à cette nouvelle activité le principe qui fait sa force : la maitrise du processus de fabrication, depuis l'élaboration du métal, jusqu'au produit fini, en l'occurrence, des avions de guerre, bombardiers comme chasseurs, et quelques modèles à usage civil. Mais l'expérience ne va guère au-delà de la conception d'appareils certes très innovants, mais lourds et rigides. Dès lors, les établissements Schneider cantonnent leur présence dans les alliages légers à la fourniture d'Alférium, notamment sous la forme d'ébauches d'hélices, ainsi que de tôles, de bandes et de profilés. Dans ce périmètre restreint, malgré une concurrence acharnée, l'entreprise devient un partenaire incontournable des principaux acteurs de la filière aéronautique française.

During the World War I, Schneider \& Co is confronted with several requests which lead them to take an interest in the question of light alloys, based on aluminium. With their skills in the development of special steel, due to the existence of a metallurgical laboratory of excellence at Le Creusot, the company managed to register its own brand in 1923: the Alferium. At the same time, it decided to apply the principle that makes its strength: control of the manufacturing process, from the elaboration of the metal to the final product (warplanes and civil planes). But the experience hardly goes beyond the design, the device being certainly innovative but heavy and rigid. That is why Schneider factories decided to restrain their activity to the Alferium's production, especially propellers, sheet metal strips and sections. Despite a very strong competition, the company became a major partner for the French aeronautic industry.

\section{INDEX}

Mots-clés : histoire des techniques, histoire des entreprises, entreprise, armement, guerre, alliage, acier, aluminium, aviation, métallurgie

Keywords : history of technology, business history, industry, alloy, steel, warfare, war, aluminium, aviation, metallurgy

\section{AUTEUR}

\section{JEAN-PHILIPPE PASSAQUI}

Jean-Philippe Passaqui est chercheur affilié à l'IHMC (UMR 8066, Paris I Panthéon-Sorbonne-ENS Ulm-CNRS). Agrégé d'histoire et docteur en histoire contemporaine, il est spécialiste d'histoire des entreprises et de l'industrie. Il a notamment publié La Stratégie des Schneider en 2006 (réed. 2015) et Les voyages forment l'ingénieur, Paris, Classiques Garnier, en 2015. Avec Marco Bertilorenzi et Anne-Françoise Garçon, il a dirigé les actes du colloque Entre technique et gestion, une histoire des ingénieurs civils des mines, Paris, Presses des Mines, 2016 et avec Marco Bertilorenzi et Nadine Dubruc, l'ouvrage Henri Fayol, les multiples facettes d'un manager, Paris, Presses des Mines, 2019. Sous le parrainage d'Anne-Françoise Garçon, il prépare actuellement son HDR. 